\title{
Three dimensional structure of a regular sunspot from the inversion of IR Stokes profiles
}

\author{
S. K. Mathew ${ }^{1}$, A. Lagg ${ }^{1}$, S. K. Solanki ${ }^{1}$, M. Collados ${ }^{2}$, J. M. Borrero ${ }^{1}$, S. Berdyugina ${ }^{3}$, \\ N. Krupp ${ }^{1}$, J. Woch ${ }^{1}$, and C. Frutiger ${ }^{3}$ \\ 1 Max-Planck-Institut für Aeronomie, 37191 Katlenburg-Lindau, Germany \\ e-mail: lagg@linmpi.mpg.de, solanki@linmpi.mpg.de, borrero@linmpi.mpg.de, krupp@linmpi.mpg.de, \\ woch@linmpi.mpg.de \\ 2 Instituto de Astrofísica de Canarias, La Laguna, Tenerife, Spain \\ e-mail: mcv@ll.iac.es \\ 3 Institut für Astronomie, ETH, 8092 Zürich, Switzerland \\ e-mail: sveta@astro.phys.ethz.ch, frutiger@astro.phys.ethz.ch
}

Received 7 May 2003 / Accepted 8 August 2003

\begin{abstract}
The magnetic, thermal and velocity structure of a regular sunspot, observed close to solar disk center is presented. Spectropolarimetric data obtained with the Tenerife Infrared Polarimeter (TIP) in two infrared Fe I lines at $15648.5 \AA$ and $15652.8 \AA$ are inverted employing a technique based on response functions to retrieve the atmospheric stratification at every point in the sunspot. In order to improve the results for the umbra, profiles of Zeeman split OH lines blending the Fe I $15652.8 \AA$ are also consistently fit. Thus we obtain maps of temperature, line-of-sight velocity, magnetic field strength, inclination, and azimuth, as a function of both location within the sunspot and height in the atmosphere. We present these maps for an optical depth range between $\log \tau_{5}=0$ and $\log \tau_{5}=-1.5$, where these lines provide accurate results. We find decreasing magnetic field strength with increasing height all over the sunspot, with a particularly large vertical field gradient of $\sim-4 \mathrm{G} \mathrm{km}^{-1}$ in the umbra. We also observe the so called "spine" structures in the penumbra, i.e. extended radial features with a stronger and more vertical magnetic field than the surroundings. Also we found that the magnetic field zenith angle increases with height. From the velocity map it is clear that the Evershed flow avoids the spines and mostly concentrates in the more inclined intervening field. The field inclination at a few locations in the outer penumbra in lower layers goes beyond $90^{\circ}$. These locations coincide with the strongest flows in the velocity map.
\end{abstract}

Key words. Sun: sunspots - Sun: magnetic fields - Sun: infrared - Sun: general

\section{Introduction}

The magnetic field at the solar surface manifests itself most prominently in the form of sunspots. In spite of significant advances in observations of the sunspot magnetic structure (e.g. Degenhardt \& Wiehr 1991; Title et al. 1993; Lites et al. 1993; Keppens \& Martínez Pillet 1996; Rüedi et al. 1998, 1999; Schlichenmaier \& Schmidt 2000; Westendorp Plaza et al. 1997a, 2001a,b) a completely coherent picture still has not emerged (Solanki 2003). A well established observational description of the vector magnetic field and thermal structure could greatly benefit the sunspot modeling efforts. Considerable advances have come from the application of inversion techniques, where one obtains the atmospheric stratification of various physical parameters by fitting synthetic to observed Stokes profiles. Recent inversion codes utilize the full information given by the spectrally resolved Stokes profiles, to

Send offprint requests to: S. K. Mathew,

e-mail: shibu@ll.iac.es infer the physical state of the atmosphere (Ruiz Cobo \& Del Toro Iniesta 1992; Frutiger et al. 2000; Westendorp Plaza et al. 2001a) and thus allow the three-dimensional structure of the atmosphere to be inferred.

Infrared lines of $\mathrm{Fe}_{\mathrm{I}}$ at $1.56 \mu \mathrm{m}$ have proved to be a good tool for probing the magnetic field in various solar features. The Zeeman splitting relative to the line width is approximately three times larger at this wavelength compared to the visible, making these lines more sensitive to weaker fields. Also, compared with visible lines, the contamination due to scattered light is much smaller in the $H$-band (Kopp \& Rabin 1992). In this paper we use spectropolarimetric measurements in two neighboring $\mathrm{Fe}_{\mathrm{I}}$ lines $(15648.5 \AA$, and $15652.8 \AA)$ in the IR $H$-band. These lines are formed deeper in the atmosphere (about $110 \mathrm{~km}$ above the base of the quiet photosphere, Bruls et al. 1991) than most visible lines. A detailed discussion about the usefulness of this set of lines to probe solar magnetic fields can be found in Solanki et al. (1992a). These lines were earlier used for studies of the thermal-magnetic 


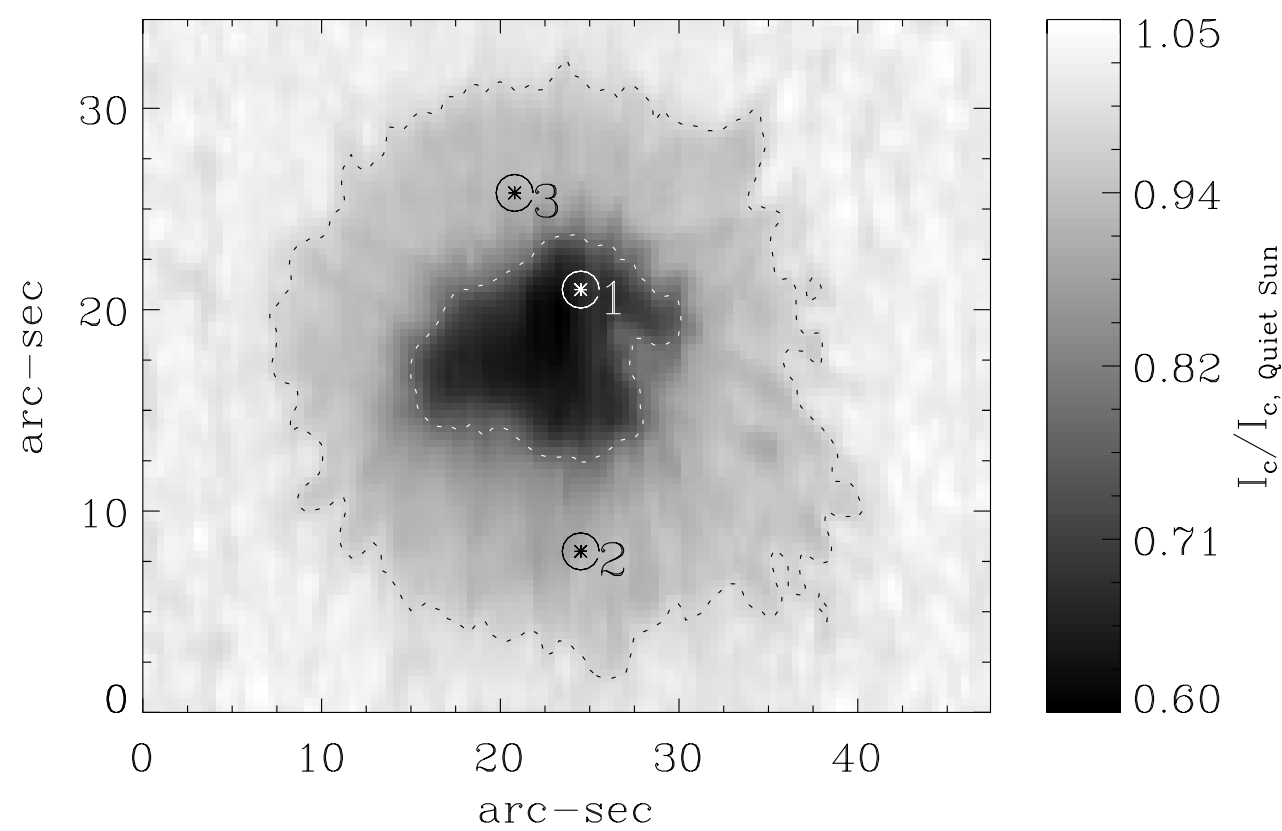

Fig. 1. Continuum intensity image of the observed sunspot. The white contour marks the umbral-penumbral, the dark contour the penumbralquiet Sun boundaries. The intensity is normalized to the average quiet Sun intensity. The asterisks symbol inside the circle show the locations of the umbral (1), penumbral (2) and neutral-line (3) profiles shown in Fig. 6.

relationship in sunspots (Livingston 1991, 2002; Kopp \& Rabin 1992; Solanki et al. 1993), magnetic field in the intranetwork (Lin 1995; Solanki et al. 1996; Lin \& Rimmele 1999; Khomenko et al. 2003) and in network regions and plages (Harvey 1977; Sun et al. 1987; Muglach \& Solanki 1992; Rüedi et al. 1992a; Bellot Rubio et al. 2001), to detect the siphon flow across neutral line of an active region (Rüedi et al. 1992b), to derive the structure of sunspots and the associated magnetic canopies (Solanki et al. 1992b; Bellot Rubio 2003) and to detect sunspot umbral magnetic field strength and velocity oscillations (Bellot Rubio et al. 2000). Recently this set of lines were used for the observational confirmation that the Evershed flow is directed along the magnetic field lines (Bellot Rubio et al. 2003). For sunspots, studies using these lines have in the past been restricted to a few spatial slices. Also, current inversion techniques provide the vertical structure in the spot. These were not available at the time that earlier studies were carried out.

In this paper, we describe the results obtained from the inversion of a data set recorded in a sunspot in the above mentioned IR lines. In the following section we describe the observational data. In the third section we deal with the data analysis technique, and present the results of some tests of the inversion procedure. In Sect. 4 we present the results of the data inversion. We discuss our results and compare them with earlier findings in Sect. 5.

\section{Observations and data reduction}

The observations were made with the Tenerife Infrared Polarimeter (TIP, Collados 1999; Martínez Pillet et al. 1999) in conjunction with the German Vacuum Tower Telescope (VTT). We recorded full Stokes profiles simultaneously of two IR Fe I lines (15648.5 $\AA, g=3$ and $15652.8 \AA, g$ eff $=1.53$ ) across a fairly round sunspot on 27 Sept. 1999, when it was near disk center $(\mu=0.92)$. The observed sunspot belonged to the region NOAA 8706 and had a maximum dimension of around $31^{\prime \prime}$. The whole scan of 172 steps with $\sim 0.4^{\prime \prime}$ step size covered a region of $68^{\prime \prime} \times 37^{\prime \prime}$, which covers the sunspot and some surrounding area.

Figure 1 shows the continuum image of the observed sunspot. The spot is fairly round with a slightly irregular umbra. Continuum intensities are obtained from the mean values of Stokes $I$ profiles over a line-free window near Fe I $15648.5 \AA$. The white and dark contours in the figure represent the umbral and penumbral boundaries, respectively. The umbral boundary encloses points with $I_{\mathrm{c}} / I_{\mathrm{c} \text {, QuietSun }} \leq 0.78$ while the penumbra includes points with $0.78<I_{\mathrm{c}} / I_{\mathrm{c} \text {, QuietSun }} \leq$ 0.9. $I_{\mathrm{c}, \text { QuietSun }}$ is the average continuum intensity of all the points, where the polarization signal $P=\left[\left(Q^{2}+U^{2}+\right.\right.$ $\left.\left.V^{2}\right) / I^{2}\right]^{1 / 2}<10^{-3}$, integrated over the observed spectral range. In Fig. 1 the slit is placed in the vertical direction and the spot is scanned from left to right.

The observations were carried out with a spectral sampling of $29 \mathrm{~m} \AA$, and covered a spectral range of $7 \AA$. The good and uniform seeing conditions during the observing interval kept the image blurring low. From the average photospheric power spectrum, we estimated the spatial resolution to be in the range of $\sim 1^{\prime \prime}-1.2^{\prime \prime}$. The correlation tracker installed at the VTT (Ballesteros et al. 1996) was used to stabilize the image, which allowed a smooth scanning throughout the observing run.

The TIP instrument uses Ferro-electric liquid crystal (FLC) retarders as polarization analyzer. A calcite beam displacer is used to divide the orthogonal polarizations, which are then imaged on a single detector array. The detector is a $256 \times 256$ liquid nitrogen cooled NICMOS-3 infrared array which is sensitive from $1 \mu \mathrm{m}-2.5 \mu \mathrm{m}$ and has a quantum efficiency better than $40 \%$ in the whole spectral range (Collados et al. 1997). 


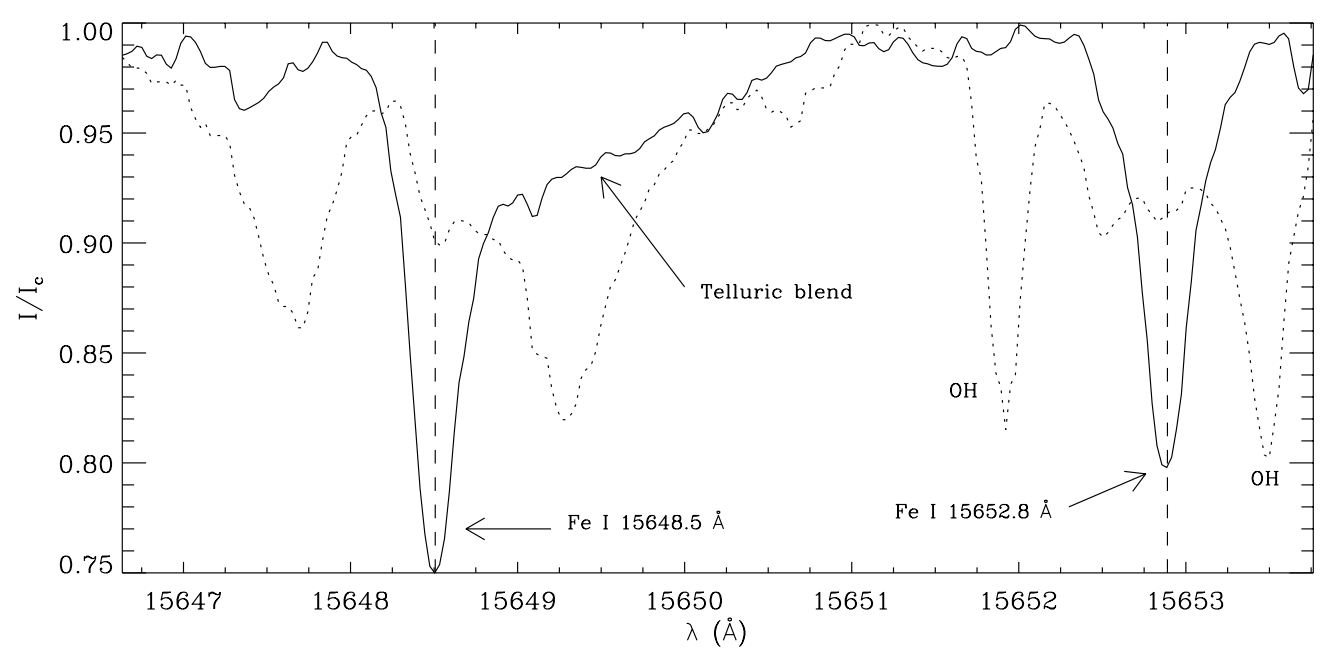

Fig. 2. Sample Stokes $I$ profiles for a quiet Sun (solid) and umbral (dotted) point. The umbral profile clearly shows the OH lines blending with the Fe I $15652.8 \AA$.

A linear combination of four measurements are made to produce the Stokes $I, Q, U$ and $V$ profiles (Bellot Rubio et al. 2000). The integration time for each accumulation and the number of accumulations can be set in accordance with the desired signal-to-noise $(\mathrm{S} / \mathrm{N})$ ratio. We use $50 \mathrm{msec}$ integration time and 10 accumulations for each image, which takes $\sim 5 \mathrm{~s}$, so that the whole scan requires $\sim 15 \mathrm{~min}$. This enables us to obtain a noise level $<10^{-3}$ in units of continuum intensity, which implies a typical $\mathrm{S} / \mathrm{N}$ of $350-400$ for the $I / I_{\mathrm{c}}$ profiles, and 200 250 for the $Q / I_{\mathrm{c}}, U / I_{\mathrm{c}}$, and $V / I_{\mathrm{c}}$ profiles in the sunspot. The flat-field, calibration and dark current measurements were performed before and after the scan, and the measurements were corrected for each of the above effects. The calibration optics allow us to remove most of the cross talk between the Stokes profiles. The residual cross-talk from $I$ to $Q, U, V$ is derived from the continuum level of the respective Stokes profiles, while the correction for $V$ to $Q, U$ and $Q, U$ to $V$ are obtained from linear regressions between the small-amplitude linear and large-amplitude circular profiles (Collados 2003).

Figure 2 shows reduced Stokes $I$ profiles for a quiet Sun (solid line) and an umbral point (dashed line). Along with the two candidate $\mathrm{Fe}$ I lines at $15648.5 \AA$ and $15652.8 \AA$, molecular and telluric blends associated with these lines are also visible in the plot. The line parameters of the two $\mathrm{Fe}_{\mathrm{I}}$ lines are given by Solanki et al. (1992a). The Fe I line at $15648.5 \AA$ has a Landé factor $g$ of 3 and is almost three times more Zeeman sensitive than the $g=3 \mathrm{Fe}_{\mathrm{I}}$ line at 5250.2 $\AA$. This IR line is completely split for field strengths greater than $500 \mathrm{G}$. Another $\mathrm{Fe}_{\text {I line at }} 15647.3 \AA$ is also visible in the figure. We have not included this line in our inversions, but in future applications it may be worthwhile considering it because it blends the Fe I line at $15648.5 \AA$ and could also provide some additional information.

Telluric and molecular blends associated with the above lines (evident in Fig. 2) introduce inaccuracies in the inversions if left unattended. We have removed the telluric blend from the Fe I $15648.5 \AA$ line by fitting the averaged quiet Sun profile, with a computed profile. The fitted profile is used to recover the blend which is subsequently removed from the other intensity profiles. Two lines of the $(3,1)$ band of the $\mathrm{OH}$ molecule (at $15651.895 \AA$ and $15653.478 \AA$ ), which are very prominent in the sunspot umbra, are blended with Fe I $15652.8 \AA$. These lines belong to Meinel system and are excited within the ground electronic state between upper and lower vibrational levels 3 and 1 and rotational levels 5.5 and 6.5. The two lines represent a $\Lambda$-type doublet arising due to the transition in the $P_{1}$ rotational branch (see details about infrared $\mathrm{OH}$ lines in Berdyugina \& Solanki 2001).

\section{Inversion technique and tests}

The data were inverted using the code "SPINOR" described by Frutiger et al. (2000). This code incorporates the "STOPRO" routines (Solanki 1987), which compute synthetic Stokes profiles of one or more lines upon input of their atomic data and one or more model solar atmospheres. LTE (Local Thermodynamic Equilibrium) conditions are assumed and the Unno-Rachkovsky radiative transfer equations (RTE) are solved. Starting with an initial guess model, the synthetic profiles were iteratively fitted to observed data using response functions (RFs) and the Levenberg-Marquardt (Press et al. 1992) algorithm to minimize the merit function $\chi^{2}$ (Ruiz Cobo \& Del Toro Iniesta 1992; Frutiger 2000). Response functions (RFs) are used for an efficient computation of derivatives of the merit function with respect to the free parameters (Landi Degl'Innocenti \& Landolfi 1982; Ruiz Cobo \& Del Toro Iniesta 1992). The RFs considerably accelerate the iterative scheme, since the $\chi^{2}$ derivatives can be obtained with a single integration of the RTE. Response functions also provide a measure of how strongly a part of a line profile is sensitive to a given physical parameter at different heights in the atmosphere.

In Figs. 3 to 5 we plot the RFs of the Stokes profiles to various atmospheric parameters. RFs represent the modification of the Stokes profiles resulting from a unit perturbation of the chosen physical parameters at the chosen optical depth. The RFs are calculated for the Stokes profiles synthesized from a typical umbral atmosphere. The atmosphere used for the synthesis is shown in Fig. 7 (top four frames), and is retrieved from the 

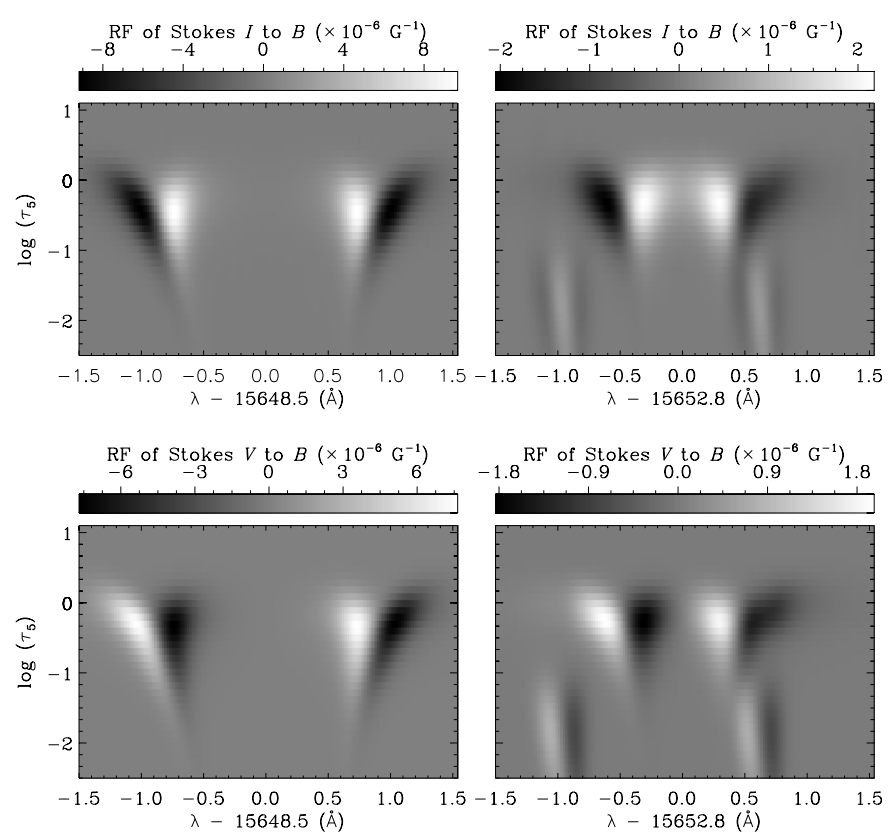

Fig. 3. Response functions of Stokes $I$ and $V$ (gray scale) to the magnetic field strength $(B)$ as a function of wavelength and logarithmic optical depth, evaluated for the umbral atmosphere shown in Fig. 7.

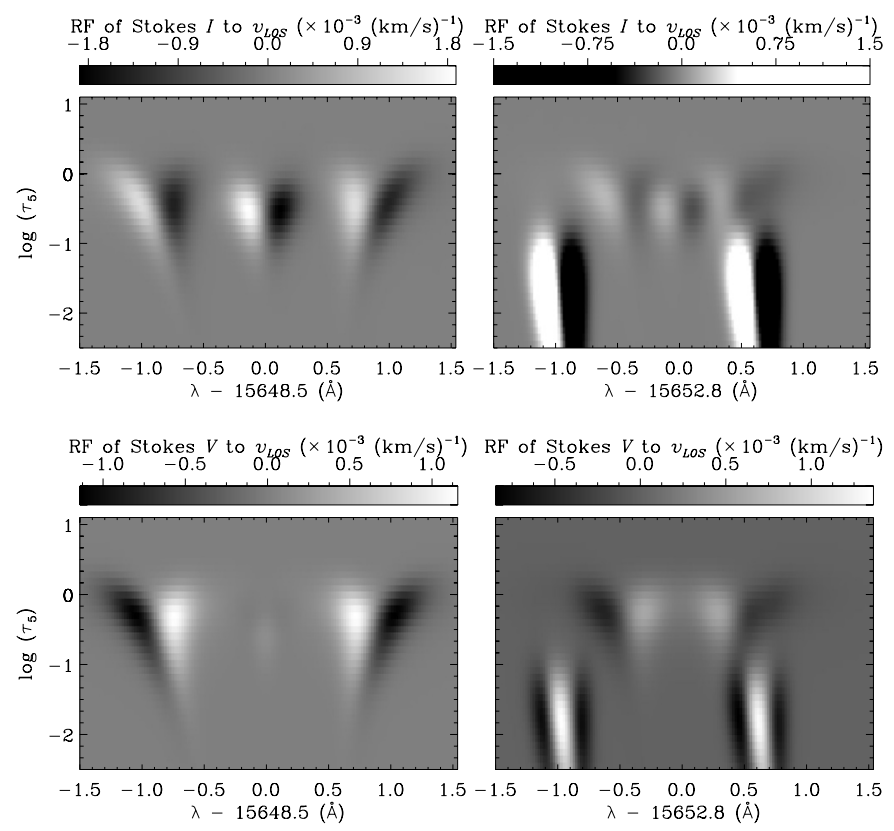

Fig. 4. Response functions of Stokes $I$ and $V$ (gray scale) to the LOS velocity $\left(v_{\mathrm{LOS}}\right)$ as a function of wavelength and logarithmic optical depth, evaluated for the umbral atmosphere shown in Fig. 7.

observed umbral profiles plotted in Fig. 6 (top set of frames). The presence of magnetic field and velocity gradients in the model atmosphere underlying these calculations can be seen from the plotted response functions (note, e.g., the shift with $\log \tau$ of the wavelength of the $\mathrm{OH}$ lines).

Figure 3 exhibits the sensitivity of the Stokes $I$ and $V$ parameters to the variations in magnetic field strength at different optical depths. For this umbral profile, the highest response for both the Fe I lines lies around logarithmic optical depth
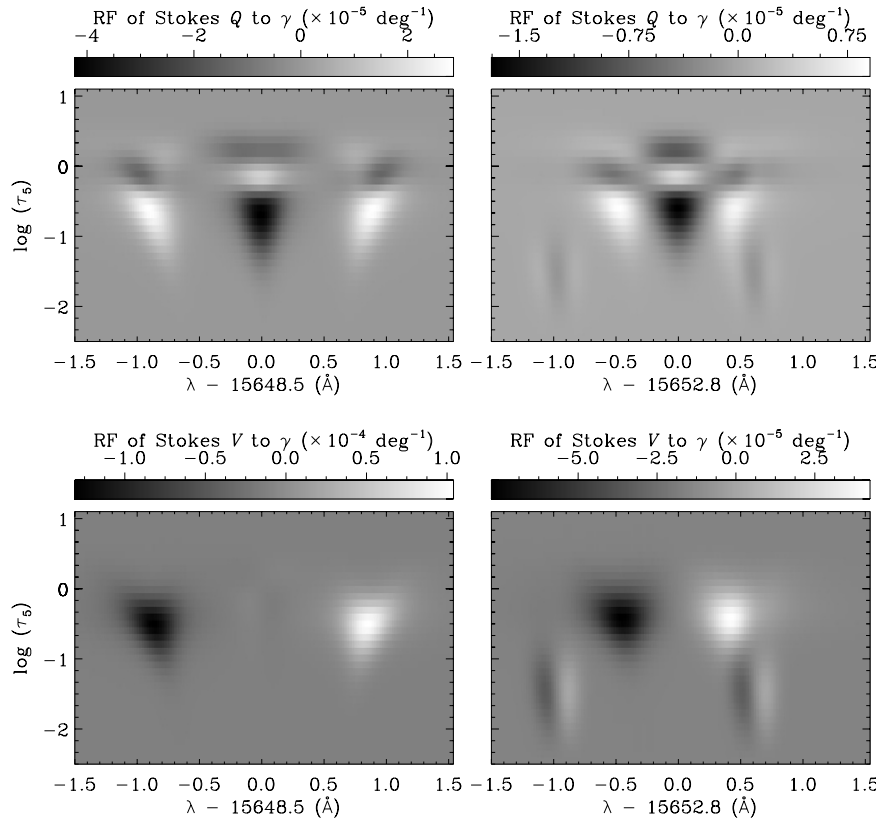

Fig. 5. Response functions of Stokes $Q$ and $V$ (gray scale) to magnetic field inclination $(\gamma)$ as a function of wavelength and logarithmic optical depth, evaluated for the umbral atmosphere shown in Fig. 7.

$\log \tau_{5}=-0.5$. At higher layers, above $\log \tau_{5}=-1.5$ the response of Stokes $I$ and $V$ to the variation in field strength is negligibly small. We also included the $\mathrm{OH}$ lines blended with Fe I $15652.8 \AA$ while computing the RFs. The low formation temperature of the $\mathrm{OH}$-molecules makes these lines more sensitive to higher and cooler atmospheric layers.

Figure 4 shows the response of Stokes $I$ and $V$ profiles to the change in line-of-sight velocity. It is noticeable that the sensitivity of the $\mathrm{OH}$ lines to the variation in velocity is much higher than that of the nearby Fe I line. This is due to the fairly large depth of the $\mathrm{OH}$-lines in the umbra. The height range over which the lines are sensitive to velocity is about the same as for $B$ (and even higher if the $\mathrm{OH}$ lines are employed). In Fig. 5 we plot the RFs of Stokes $Q$ and $V$ to the variation in the field inclination. A comparison of the three figures shows that each atmospheric parameter produces its own distinct pattern, allowing them to be distinguished from each other (cf. Ruiz Cobo \& Del Toro Iniesta 1994). In general, much of the contribution to the RFs of various Stokes parameters to different atmospheric parameters results from a relatively narrow optical depth range, which spans deeper layers than typical lines in the visible are sensitive to $\left(\log \tau_{5}=0\right.$ to $\left.\log \tau_{5}=-1.5\right)$.

In our inversions the Stokes profiles were calculated in LTE through a two-component model atmosphere: in every pixel two atmospheric components are allowed for, one magnetic (with a filling factor $\alpha$ ) and one field-free (with filling factor $1-\alpha), \alpha$ is a free parameter in the inversions. The field-free component describes the non-magnetic stray light contamination in the spectrum and the filling factor $\alpha$ is a measure of the stray light contamination in the profiles.

We are aware that at least in the penumbra this approach represents a considerable simplification, given that the magnetic and thermal structure is extremely inhomogeneous 

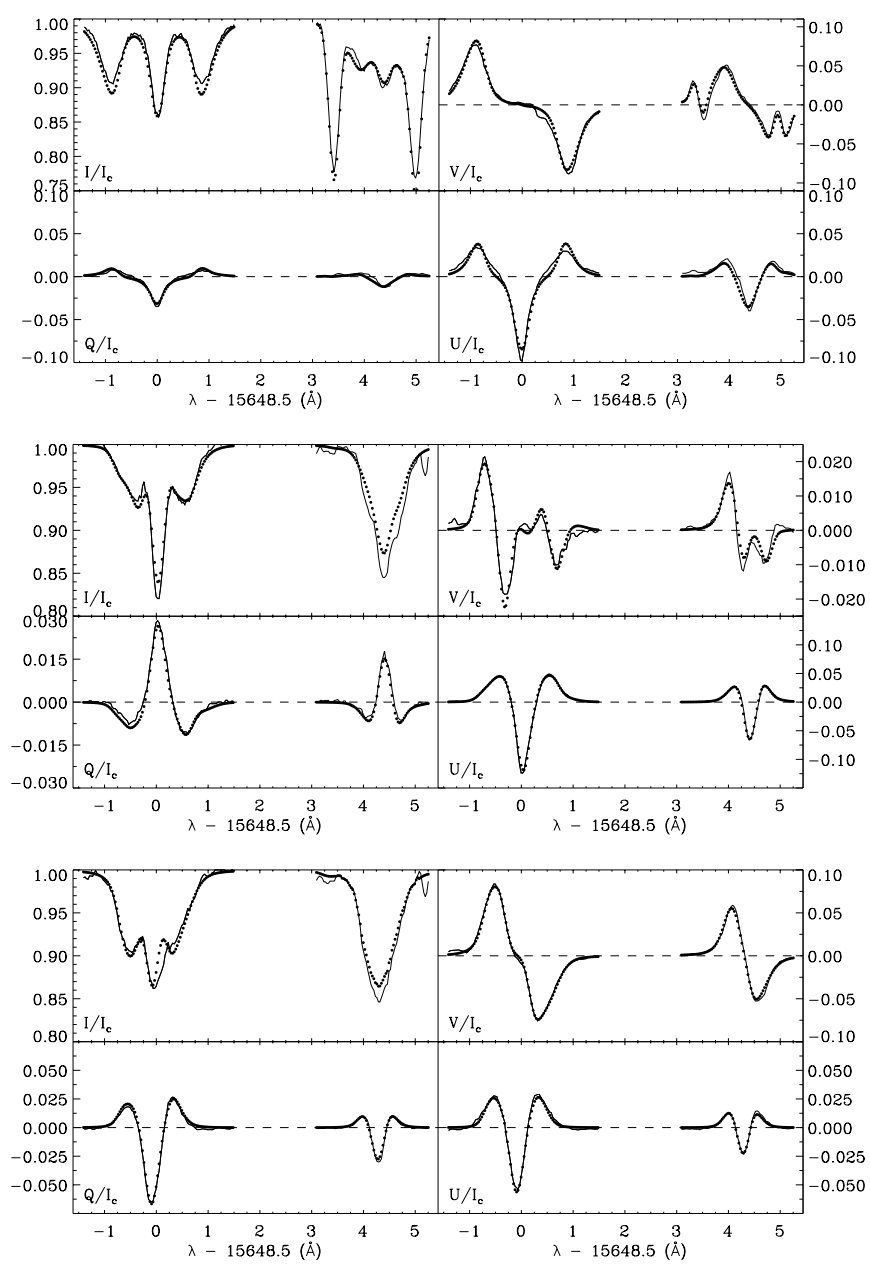

Fig. 6. Typical fits obtained from the inversion (dots) of Stokes profiles observed (solid curves) at an umbral location (top 4 frames), at the neutral line in the limb-side penumbra (middle 4 frames) and at a point in the center-side penumbra (bottom 4 frames).

(e.g. Degenhardt \& Wiehr 1991; Schmidt et al. 1992; Title et al. 1993; Solanki \& Montavon 1993; Martínez Pillet 2000; Del Toro Iniesta et al. 2001; Schlichenmaier \& Collados 2002). Recently Bellot Rubio (2003) presented the inversion results of a similar data set. The models used for his analysis include atmospheres with one and two magnetic components. From the analysis he concludes that increasing the complexity of the model allows the properties of the unresolved structure of the sunspot penumbra to be deduced. However, the general configuration of the vector magnetic field and the flow field can be investigated with the help of a simple one-component model, which forms the first step of a more detailed investigation involving concrete models of penumbral fine structure. There is a rich literature on the magnetic structure of sunspots deduced in this manner (see Solanki 2003 for a review). Thus the present investigation is an IR counterpart to earlier studies based on data obtained in the visible spectral range (e.g. Westendorp Plaza et al. 2001a, 2001b).

We cautious that, vertical gradients may not be reliably determined when discontinuities along the line-of-sight exist (as have been proposed by, e.g. Solanki \& Montavon 1993; Martínez Pillet 2001). Thus it is the general behavior of the
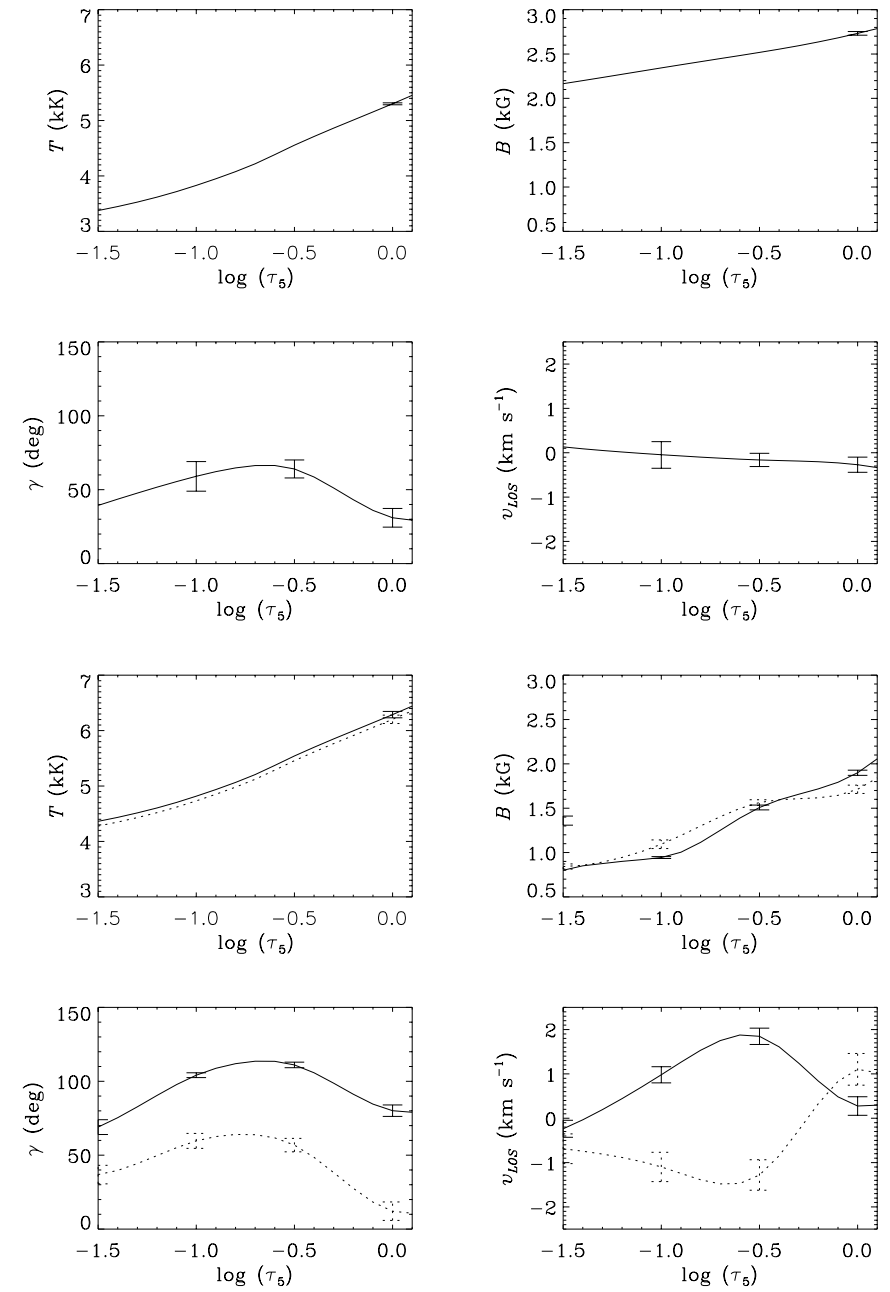

Fig. 7. Atmospheric stratification obtained from the profiles shown in Fig. 6. Top 4 frames: for the umbral point. Bottom 4 frames: for the point at the neutral line (solid lines) and for the center-side penumbral point (dotted lines). Bars indicate the formal errors of the inversion at the nodes. $\gamma$ denotes the field inclination with respect to the line-of-sight and positive values of the LOS velocity indicate redshifts.

atmospheric parameters which is well determined, not the exact numbers returned by the inversion code. Also, the azimuthal fluctuations of the atmospheric parameters indicated by simple one component inversions might be an artifact of the model in the presence of azimuthal variations of the fraction of the lineof-sight and/or resolution element filled by horizontal penumbral tubes (Martínez Pillet 1997; Bellot Rubio 2003).

The free parameters in the inversion are temperature $(T)$, magnetic field strength $(B)$, field inclination in the line-of-sight reference frame $(\gamma)$, field azimuth $(\chi)$, line-of-sight velocity $\left(v_{\mathrm{LOS}}\right)$, micro- $\left(\xi_{\mathrm{mic}}\right)$ and macro-turbulent $\left(\xi_{\mathrm{mac}}\right)$ velocities. The free parameters are specified at 6 nodes $^{1}$ at fixed logarithmic optical depth for the magnetic component, except for temperature, micro-, and macro- turbulent velocities. The microand macro-turbulent velocities are assumed to be constant with

\footnotetext{
1 Nodes are discrete locations in the optical depth scale where the iterative modification of various parameters are carried out. The modifications in between the nodes are specified by a smooth interpolation.
} 

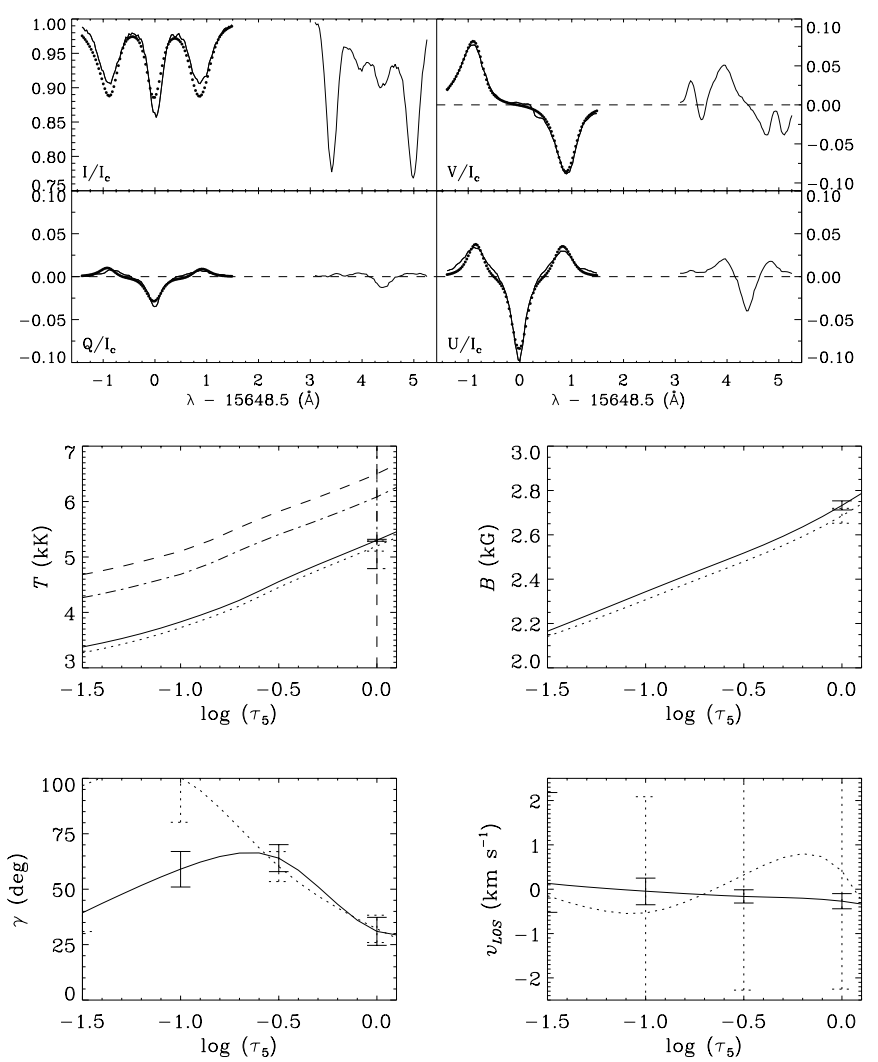

Fig. 8. Top 4 frames: fits (dots) to Stokes profiles observed (solid line) for the same umbral location as in Fig. 6, but now without including the $\mathrm{Fe}$ I line at $15652.8 \AA$ and the blending $\mathrm{OH}$ lines in the inversion. Bottom 4 frames: The atmospheric stratification returned by the inversion without fitting $\mathrm{Fe}$ I line at $15652.8 \AA$ and the blending $\mathrm{OH}$ lines (dotted lines), and when these two are included (solid lines). The respective error in the estimation of these parameters are indicated by the bars. Dashed and dotted-dashed lines in the temperature plot represent the temperature of the stray-light component with and without including the $\mathrm{OH}$ lines in the inversions, respectively. $\gamma$ denotes the field inclination with respect to the line-of-sight and positive values of the LOS velocity indicate redshifts.

depth. The temperature stratification is taken from the Kurucz (1992) radiative equilibrium model for an effective temperature $\mathrm{T}_{\mathrm{eff}}=5000 \mathrm{~K}$, but is shifted to a reference temperature specified for $\log \tau=0$, where $\tau$ is continuum optical depth at $5000 \AA$. We opted to restrict the freedom of choice of temperature stratification in view of the limited temperature sensitivity and relatively small height range of formation of these lines. For the non-magnetic component we use the Kurucz quiet Sun model. Shift of the temperature stratification of the fieldfree component representing the stray light is also taken as a free parameter.

Also, we opted for a simplified inversion of the umbral profiles, where we specify the initial guess value for the field strength only at a single node $\left(\log \tau_{5}=0\right)$ and introduce a height independent vertical gradient $(\mathrm{d} B / \mathrm{d} z)$.

The inversion procedure modifies the atmosphere iteratively until the difference between the synthetic profiles calculated with the new atmosphere and the observed ones is minimized. Figure 6 shows typical fits for three spatial points, one

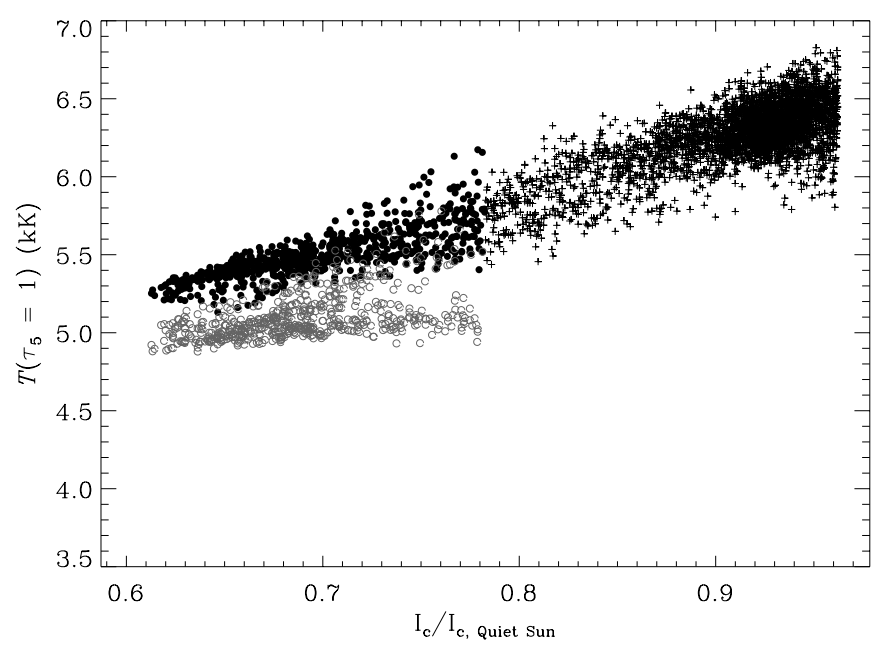

Fig. 9. Normalized continuum intensity versus temperature obtained from the inversion. Umbral points are denoted by filled circles and penumbral points by plus signs. Gray circles denote the retrieved temperature of the umbral points without fitting the Fe I line at $15652.8 \AA$ blended with molecular $\mathrm{OH}$ lines.

in the umbra and two in the penumbra. Of the later, one is at the neutral line on the limb-side, while the other is in the center-side penumbra, roughly at the same distance from the umbral boundary. Note that the irregular, multi-lobed shape of the Stokes $V$ profile at the neutral line is reasonably reproduced in spite of the simplicity of the underlying model.

Figure 7 displays the atmospheric stratifications retrieved from the inversions of these profiles. The error bars represent the formal standard errors at different nodes. For the two points in the penumbra the retrieved parameters are similar (with the exception of the absolute value of the inclination to the LOS and the LOS velocity, which need to be different of course), even though the corresponding Stokes profiles look very different. This agreement gives us some confidence that the retrieved parameters are reliable. More tests are described below.

The molecular $\mathrm{OH}$ lines blended with $\mathrm{Fe}_{\mathrm{I}} 15652.8 \AA$ are also inverted, which improves the reliability of the deduced atmospheric parameters, in particular in the umbra where these blends are strong (Berdyugina 2002). Figure 8 (top four frames) shows the fits for the same umbral point plotted in Fig. 6 without including the molecular lines in the inversion. In the bottom four frames in Fig. 8, we compare the retrieved atmospheres with (solid lines) and without (dotted) including the molecular lines in the inversions. Evidently, the retrieved parameters depend strongly on whether we include the $\mathrm{OH}$ lines in the inversions. Thus the magnetic field and velocity gradients are significantly different in the two cases. The formal errors are also significantly larger if the $\mathrm{OH}$ lines are not included. The dashed and dotted-dashed curves in the temperature plot show the retrieved temperatures for the non-magnetic component, with and without molecular lines respectively. Without $\mathrm{OH}$ lines the stray light component is cool i.e. cooler than both the quiet Sun and the penumbra, whereas with the inclusion of $\mathrm{OH}$ lines the stray light component becomes as hot as the photosphere. The filling factors differ also in the two cases. 

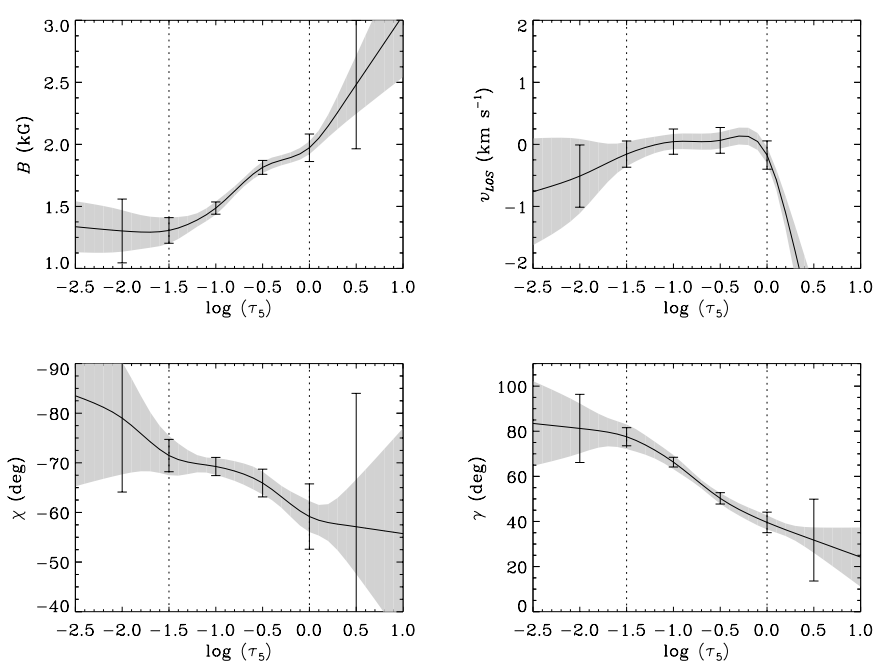

Fig. 10. Atmospheric stratification of field strength $B$, line-of-sight velocity $v_{\mathrm{LOS}}$, field azimuth $\chi$, and line-of-sight field inclination $\gamma$. The shaded area indicates the standard deviation of these parameters for inversions starting from different initial values. Error bars indicate the average formal errors returned by one such inversion (solid lines). The values within the optical depth range enclosed by the vertical dotted lines are used to produce the maps of various parameters.

As a further illustration of the reliability of the inversions and the importance of fitting the $\mathrm{OH}$ lines, we display the scatter plot for the temperature retrieved from the inversions (for the $\log \tau_{5}=0$ layer) and the continuum intensity in Fig. 9. Since we invert spectra normalized to the local continuum intensity, the temperature values obtained by the two techniques are completely independent of each other. The filled circles and plus signs represent the retrieved temperature of umbral and penumbral points, respectively. Low scatter in the umbra is particularly attributed to the temperature sensitivity of the $\mathrm{OH}$ lines, which are strong there. For comparison we plot the gray circles which denote the temperature retrieved for the same umbral points without fitting the second $\mathrm{Fe}_{\mathrm{I}}$ line (at $15652.8 \AA$ ) which is blended with $\mathrm{OH}$ lines. Not only is the scatter larger, but the $\mathrm{Fe}_{\mathrm{I}}$ lines on their own tend to systematically underestimate the umbral temperature.

To further evaluate the consistency of the physical parameters of the sunspot atmosphere retrieved by the inversion code from the observed Stokes profiles, we have carried out tests on observed Stokes profiles with different initialization values for various physical parameters in the initial guess model. Figure 10 exhibits the result of one such test for an inner penumbral point. For the tests we have taken 15 random initialization values each for temperature (within 4000 to $7000 \mathrm{~K}$ ), magnetic field (within 0 to $2500 \mathrm{G}$ ), field inclination (within 10 to 90 degrees) and line-of-sight velocity (within the range -2 to $2 \mathrm{~km} \mathrm{~s}^{-1}$ ). The shaded region shows the standard deviation of the parameters retrieved using various initializations and the error bars represent the average formal errors in these parameters returned independently by the code. The standard deviations of the retrieved parameters are smaller than the average formal errors, at least for the optical depth range between $\log \tau_{5}=$ 0 and $\log \tau_{5}=-1.5$. This range agrees well with the expected range over which these lines are sensitive to atmospheric parameters (Figs. 3-5). In the following sections, we therefore discuss the results obtained only for this depth range where the inversion results are reasonably reliable.

\section{Inversion results}

In this section we present the inversion results for a part of the observed area which includes the sunspot and its immediate surroundings $(32 \mathrm{Mm} \times 28 \mathrm{Mm})$. We convert the retrieved parameters from the line-of-sight (LOS) frame to the local solar frame using the method described in Hagyard (1987), cf. Venkatakrishnan et al. (1988). By doing so we obtained vertical $\left(B_{z}\right)$ and radial $\left(B_{r}\right)$ components of the magnetic field and also the corrected azimuth $(\psi)$ and field inclination, with respect to the solar surface normal, denoted hereafter as the zenith angle, $\zeta$. We have not applied any correction to the derived line-of-sight velocities. Since the wavelength calibration is carried out using the quiet Sun profiles, all the line-of-sight velocities are retrieved by the inversions with respect to the quiet Sun photosphere.

We specifically concentrate on the layers ranging from logarithmic depths $\left(\log \tau_{5}\right) 0$ to -1.5 . This is found to be the most reliable range, where the lines have considerable response to the change in physical parameters (see Sect. 3) and the errors in our inversion results are small. The results are presented as three slices by averaging from $\log \tau_{5}=0$ to -0.5 , from -0.5 to -1 and from -1 to -1.5 . The averaging reduces some fluctuations in vertical gradients of some physical quantities. Since we are more interested in the general behavior of such gradients rather than their exact values (a more sophisticated model is required for that) this approach appeared to be reasonable. Only the pixels with a magnetic filling factor $(\alpha)$ larger than 0.05 are plotted.

\subsection{Maps of physical parameters}

Figure 11 shows the results for the field strength $(B)$, the vertical $\left(B_{z}\right)$, and the radial $\left(B_{r}\right)$ components of the field, for the three vertically stacked $\tau$ slices described above. In Fig. 12 we display the maps for the field zenith angle $(\zeta)$, field azimuth $(\psi)$, and the line-of-sight velocity $\left(v_{\mathrm{LOS}}\right)$ for the same layers. The continuum intensity contours marking the umbral and penumbral sunspot boundaries are over-plotted on the figures. The arrow points towards solar disk center.

The irregular outline of the umbra in the continuum image is to a certain extent reflected in the field strength map. In lower layers, the strongest fields concentrate in locations closer to the center of the umbra. Another, somewhat less strong field concentration is found in a small area near the umbral boundary (at around $20 \mathrm{Mm}$ in the horizontal and $16 \mathrm{Mm}$ in the vertical direction), where the umbra slightly protrudes into the penumbra. The maximum field strength found in this spot is around $2800 \mathrm{G}$ near the center of the umbra, which coincides with the darkest region in the umbra. The field strength smoothly decreases in almost all directions from this point, in particular in the deeper layers (an exception is the direction in which the secondary maximum of the field strength is located), and reaches a value of around 1400-1500 G near the penumbral 

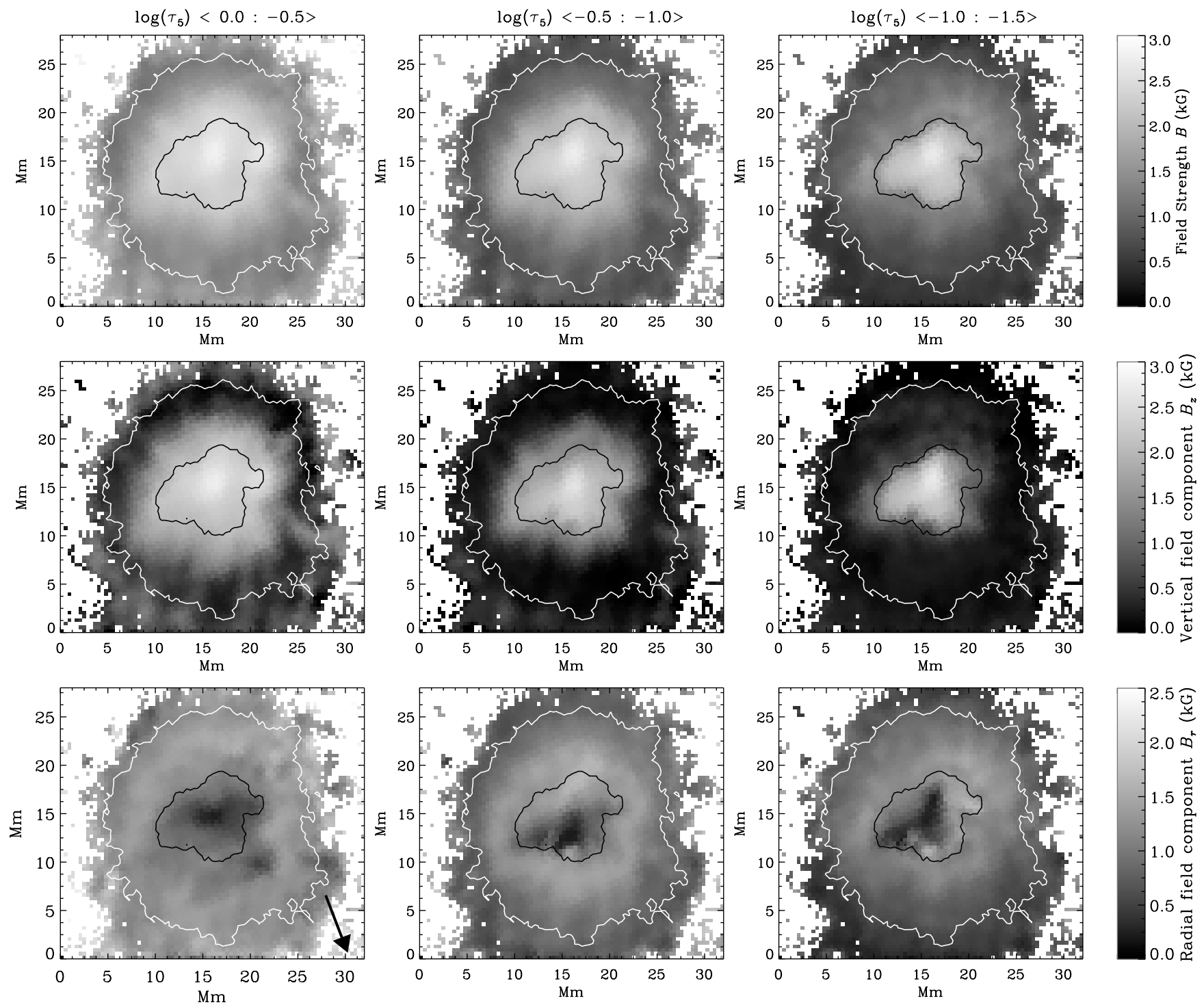

Fig. 11. Maps of the magnetic field strength $(B)$, as well as of the vertical $\left(B_{z}\right)$ and radial $\left(B_{r}\right)$ field components from top to bottom. For each parameter three maps representing the $\log \tau_{5}$ layer 0 to $-0.5,-0.5$ to -1.0 and -1.0 to -1.5 are plotted (from left to right). The contours represent the umbral and penumbral boundaries derived from the continuum intensity. The arrow in the lower-left panel marks the direction to solar disk center.

boundary in the deepest layer. The field decreases as we move to the upper layers. We found an average vertical gradient of around $-4 \mathrm{G} / \mathrm{km}$ in the umbra and a slightly higher value in the penumbra. In the coolest part of the umbra, however, the vertical $B$-gradient turns out to be considerably smaller, being less than $-1 \mathrm{G} / \mathrm{km}$. Also, in much of the penumbra the large vertical gradient is restricted to the deeper layers.

The umbral field is found to show little horizontal structure beyond the two maxima. In the penumbra, diffuse radial structures with a larger field strength are evident. Such spines of enhanced field strength are mainly visible in the lower and intermediate layers, being relatively washed out in the higher layers.

The maximum vertical field $B_{z}$ coincides with the location of maximum field strength and has almost the same strength, indicating that the strongest field also coincides with the most vertical field. The $B_{z}$ component decreases outward more rapidly than $B$. For both quantities the drop with height is more rapid in the penumbra than in the central umbra. The "spine" structures are more evident in the $B_{z}$ maps (some of these structures are marked with arrows in the zenith angle map in Fig. 13b). At least in the lower layers some of these structures are traceable until the penumbral boundary.

We found that $B_{z}$ drops below zero at a few locations along the penumbral boundary in the lower layers, corresponding to locations where $\zeta$ is greater than $90^{\circ}$ and the field points back to the Sun again. Interestingly, such points are not seen in the middle and higher layers. This is very similar to the results of Westendorp Plaza et al. (1997b).

$B_{r}$ increases radially outward from the center of the umbra until it reaches a maximum value in the central penumbra. From there it decreases again outward only very gently. The spine structure is less clearly visible in $B_{r}$, with indications that $B_{r}$ is weaker at the locations where $B_{z}$ is strong. 

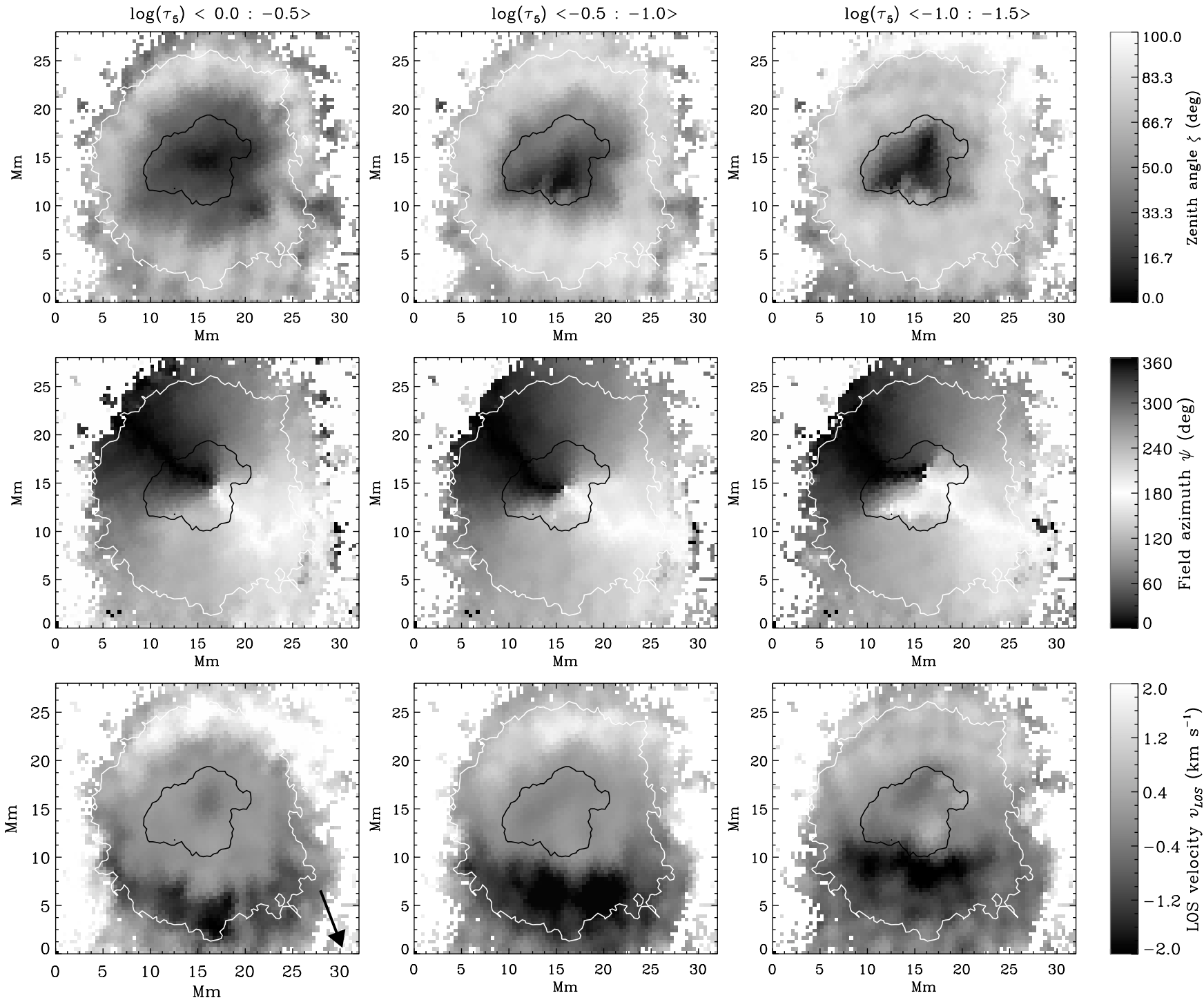

Fig. 12. Same as Fig. 5, but for the zenith angle $(\zeta)$, field azimuth $(\psi)$ and line-of-sight velocity ( $\left.v_{\text {Los }}\right)$. The velocity map is clipped to $\pm 2 \mathrm{~km} \mathrm{~s}^{-1}$ in order to enhance the contrast.

In the higher layers $B_{r}$ decreases considerably and the location of the maximum shifts inwards, in the direction of the umbralpenumbral boundary.

Figure 12 shows maps of the zenith angle of the field $(\zeta)$, the corrected azimuth $(\psi)$ and the line-of-sight velocity $\left(v_{\mathrm{LOS}}\right)$ at different layers. As expected, the inclination is near zero in the umbral core and increases outwards, although in the higher layers the inversion returns relatively similar $\zeta$ values throughout the outer penumbra. $\zeta$ reaches an average value of $70^{\circ}-80^{\circ}$ near the outer penumbra. At a few locations in the outer penumbra $\zeta$ runs beyond $90^{\circ}$ (corresponding to a negative $B_{z}$ component), although $\zeta$ is never larger than $110^{\circ}$. Hence the field lines returning to the solar surface are nearly horizontal. The inclination of the field lines increases strongly with increasing height, changing by as much as $45^{\circ}$ in the inner penumbra between the layers $\log \tau_{5}=0$ and $\log \tau_{5}=-1.5$, which corresponds to roughly $130 \mathrm{~km}$ in height. Such large gradients in $\zeta$ were introduced by Sánchez Almeida \& Lites (1992) to explain broad-band circular polarization (i.e. Stokes $V$ asymmetry) and probably are an artifact introduced by the simple one component model employed. The spines are most clearly seen in the inclination. They are most prominent in the lowest layer, where they are observed to extend beyond the sunspot boundary. They are characterized by low values of inclination.

The field azimuth (second row of Fig. 12) remains almost constant in the radial direction and shows a smooth azimuthal variation. Spine structures are not prominent in any layer of the azimuth maps.

Maps of the line-of-sight velocity ( $\left.v_{\mathrm{LOS}}\right)$ obtained from the inversion are given in the bottom panels of Fig. 12. Evershed outflow is clearly visible in the penumbra. The maximum $v_{\text {LOS }}$ retrieved is $\sim \pm 2.5 \mathrm{~km} \mathrm{~s}^{-1}$. It is reached in the lower layers, where the flow is mostly concentrated in the outer penumbra. In the upper layers, the maximum flow speeds are located increasingly closer to the umbral boundary. In general, the $v_{\text {LOS }}$ decreases with height.

There is a clear asymmetry between the $v_{\mathrm{LOS}}$ in the centerside and limb-side part of the penumbra. In the lowest layers, 

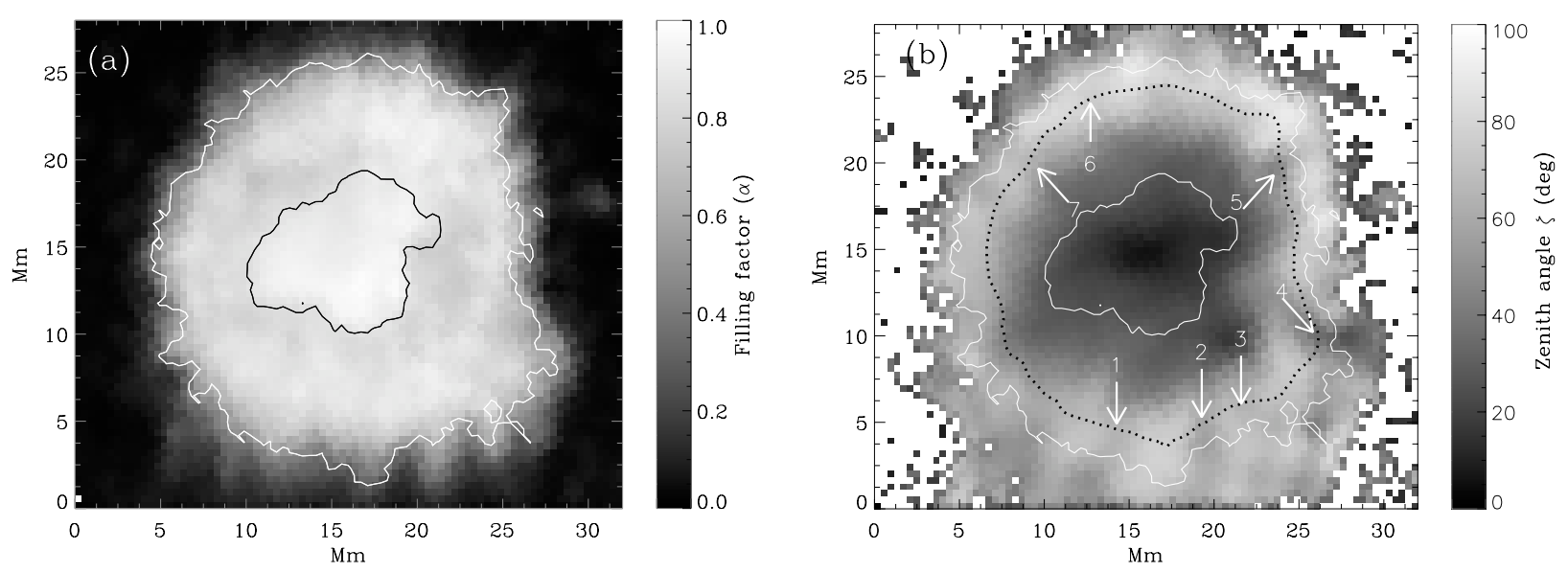

Fig. 13. a) Filling factor $\alpha$ for the magnetic component, and b) the zenith angle map for the lowest layer. The dark dotted contour in b) shows the azimuthal path considered in Fig. 14, and the arrows indicate locations identified as "spines".

the $v_{\text {LOS }}$ in the limb-side penumbra is found to be larger than in the center-side penumbra. If we assume that the penumbra on both sides of the umbra is identical and that any differences in LOS quantities are due to the different viewing angle, then this result suggests that in the deepest layers we see flow that is pointing slightly downwards (in the outer penumbra). In the higher layers (in the inner and mid penumbra), where the center-side penumbra exhibits the larger $v_{\text {LOS }}$ the outflow is pointed slightly upward (on average).

A careful comparison between the $v_{\text {LOS }}$ and the $\zeta$ maps at the lowest level reveals that the strongest Evershed speeds are mostly located in the space between "spines". Like the Evershed flow itself, this spine structure is found to extend beyond the sunspot's boundary.

Note that the flows plotted in Fig. 12 correspond to motions of magnetized material. The field-free component shows roughly similar flow speeds on both sides of the sunspot corresponding approximately to those found for the quiet Sun profiles. The strongest flow speeds in the $v_{\text {LOS }}$ maps coincide with the locations where $\zeta$ is greater than $90^{\circ}$. The errors in the retrieved $v_{\text {LOS }}$ in the umbra are much larger than in the penumbra, which could be a reason for the average residual velocity of $0.2-0.3 \mathrm{~km} \mathrm{~s}^{-1}$ found in the umbra. Since the profile in the quiet Sun granulation are blue-shifted, this relative red shift implies that the umbral gas is probably close to being at rest, in agreement with previous investigations.

Contrary to previous works (Solanki et al. 1992a; Lites et al. 1993; Westendorp Plaza et al. 2001a) we do not find any clear evidence of the sunspot canopy in the field strength maps (seen by the above authors as an increasing magnetic field strength with height beyond the visible limit of the penumbra $r / R>1)$. Although we obtain a similar behavior for a few radial slices it is not significant enough to appear in the azimuthal averages (Fig. 15, upper left panel) and on average we still find a decreasing magnetic field with height.

The magnetic filling factor plotted in Fig. 13a exhibits relatively little variation through the sunspot, although the $\alpha$ value in the penumbra is on average lower than the umbra. Considerable structure in $\alpha$ is seen just outside the sunspot's boundary. Clearly, the filling factor decreases most rapidly along the spines.

\subsection{Azimuthal cut}

In order to uncover the correlation between various parameters in the penumbra, we consider an azimuthal cut at 0.8 of the spot radius. Since the penumbra is not perfectly circular, but irregular, the cut delineates a constant $r / r_{\mathrm{s}}$ value of 0.8 (where $r_{\mathrm{s}}$ is the local sunspot radius) obtained after smoothing the intensity image with a boxcar average having a width of 6 pixels from the outer penumbral boundary (indicated by the dotted contour in Fig. 13b). Figure 14 shows the variation of $B, v_{\text {LOS }}$, $\zeta, \Delta \psi$, and $T$ along this azimuthal path for the slice covering $\log \tau_{5}>-0.5$. Here $\Delta \psi$ is the difference between the magnetic azimuth and the position angle. The vertical solid and dashed lines pass through the local maxima and minima in $\left|v_{\text {LOS }}\right|$ and $\zeta$, respectively.

A clear correspondence between lower $\left|v_{\text {LOS }}\right|$ and more vertical spine structure is seen. Similarly, the field strength often exhibits a peak where $\zeta$ is smallest. Some of these points are marked with numbered arrows, such arrows are repeated at the corresponding locations in the zenith angle map shown in Fig. 13b. We found correlation coefficients of $0.7,-0.3$ and -0.6 for $\zeta-\left|v_{\mathrm{LOS}}\right|, B-\left|v_{\mathrm{LOS}}\right|$ and $B-\zeta$, respectively for this azimuthal cut. These correlations substantiate earlier results that the Evershed flow is located mainly in regions with a nearly horizontal field, but its correlation with field strength is weak. No significant correlation is found between $T$ and other parameters. These results are in good agreement with the findings of Lites et al. (1993)

\subsection{Azimuthal averages}

Figure 15 displays the azimuthal averages for $B, B_{z}, B_{r}, \zeta, \Delta \psi$ and $v_{\mathrm{LOS}}$ for all three layers. These parameters are averaged over azimuthal paths, centered on the darkest umbral point. The vertical dotted lines represent the umbral and penumbral boundaries. The rms fluctuations of these quantities along the 

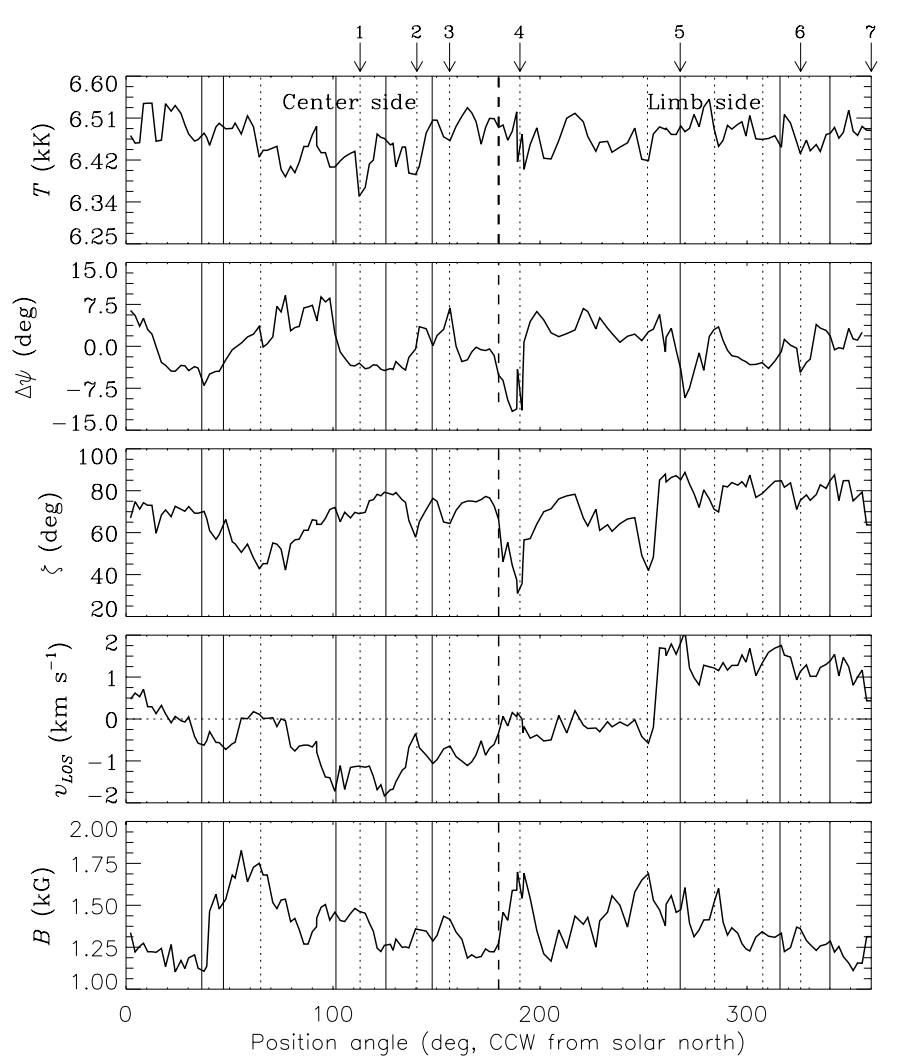

Fig. 14. Variation of $B, v_{\mathrm{LOS}}, \zeta, \Delta \psi$ and $T$ as a function of position angle along an azimuthal path in the outer penumbra for deep layers. The solid vertical and dotted lines represents local maxima in $\left|v_{\text {LOS }}\right|$ and minima in $\zeta$, respectively. The arrows point to locations exhibiting a spine structure. The thick vertical dashed line indicates position angle $180^{\circ}$.

azimuthal paths are represented by the shaded/striped areas. For $v_{\text {LOS }}$ the center-side (dotted lines)and limb-side (solid lines) velocities are plotted separately.

In all the layers we found a smooth transition of field strength at the umbral boundary. The average radial magnetic field gradient $\langle\mathrm{d} B / \mathrm{d} r\rangle$ in the umbra is found to be $\sim-0.05 \mathrm{G} \mathrm{km}^{-1}$ in the lower slice, while it increases in the upper layers $\left(\sim-0.11 \mathrm{G} \mathrm{km}^{-1}\right.$ and $\left.-0.18 \mathrm{G} \mathrm{km}^{-1}\right)$. In the penumbra the average radial gradient is $\sim-0.12 \mathrm{G} \mathrm{km}^{-1}$. The average vertical gradient $\langle\mathrm{d} B / \mathrm{d} z\rangle$ obtained for the umbra is around $-4 \mathrm{G} \mathrm{km}^{-1}$. Note that $\mathrm{d} B / \mathrm{d} z$ is rather small in the darkest part of the spot (only $-1 \mathrm{G} \mathrm{km}^{-1}$ ), but increases rapidly towards the penumbra, where it remains uniformly large.

In the upper right panel we plot the azimuthal averages for the $B_{z}$ and $B_{r}$ components. Along the radial direction $B_{z}$ decreases rapidly, with the drop being steeper in the lower layers. The radial dependence of $B_{z}$ mimics that of $B$ except that it drops more rapidly. The $B_{r}$ component steadily increases towards the penumbra. It remains constant beyond $r=0.6 r_{\mathrm{s}}$ in the lower layers, where $r_{\mathrm{s}}$ is the spot radius, while slightly dropping in the upper layers beyond the umbral-penumbral boundary. The average radial gradient for $B_{z}$ and $B_{r}$ are $-0.18 \mathrm{G} \mathrm{km}^{-1}$ and $0.04 \mathrm{G} \mathrm{km}^{-1}$, respectively.

In the lower left frame the radial dependence of $\langle\zeta\rangle$ and $\langle\Delta \psi\rangle$ is plotted. The average radial gradient deduced for $\zeta$ in the umbra is $\sim 7 \times 10^{-3} \mathrm{deg} \mathrm{km}^{-1}$. The gradient remains similar in the penumbra up to around 0.8 spot radii. The zenith angle increases with height. The azimuthal averages for the relative field azimuth $(\Delta \psi$, measured counterclockwise from solar north) are a measure of a global twist of the sunspot's field. $\Delta \psi$ shows a small variation of twist along the azimuthal direction ( $\sim 5$ degrees to $\sim+5$ degrees from the umbral boundary towards the penumbral boundary). This variation could be an artefact due to the irregular shape of the umbra. In general there is no indication of a significant twist of the sunspot's magnetic field.

The asymmetries in flow velocities described in the earlier section, between the center-side and limb-side penumbra is clear in the LOS velocity plot (lower right frame). The rms fluctuation in the velocity is plotted only for the lower slice. Note that due to the averaging over azimuth the flow speeds plotted in Fig. 15 are considerably lower than the measured peak values. Assuming a radially directed flow, which is a good approximation due to the small $\Delta \psi$, we obtain a factor of $\sim 1.6$ between peak measured and averaged value, which comes close to the actual value. The true flow speeds are in turn expected to be a factor of $1 / \sin \theta$ larger, so that the azimuthal averages needs to be multiplied by a total factor of $2.5 \times 1.6=4$ to get the true Evershed flow speed. Thus, the measured values indicate peak speeds of the horizontal flow of up to $6 \mathrm{~km} \mathrm{~s}^{-1}$.

In Fig. 16 we plot the azimuthal averages for the continuum intensity $\left(I / I_{\text {QuietSun }}\right)$ and the scattered light parameter $(1-\alpha)$. The continuum intensity increases smoothly outwards form the umbra and shows the largest gradient near the umbralpenumbral boundary, at around $0.4 r / r_{\mathrm{s}}$. The average scattered light parameter is around 0.06 (94\% filling factor for the magnetic component) in the center of the umbra, and increases to $\sim 0.5$ near the outer penumbra. A local maximum in this parameter is noticed near the umbral-penumbral boundary, where the gradient in continuum intensity is large. It, like the rapid increase toward the sunspot boundary, could be due to spatial scattered light. $(1-\alpha)$ provides an upper limit on the amount of field-free material in the sunspot. In particular in the umbra this limit is relatively tight, around $6 \%$ in the central umbra. Such a limit in the deep layers sampled by the $1.5 \mu \mathrm{m}$ lines is an important constraint on models of umbral dots and umbral energy transport (Parker 1979; Choudhuri 1986; Degenhardt \& Lites 1993a,b).

\subsection{Average models for umbra and penumbra}

In Fig. 17 we present average model atmospheres for umbra and penumbra, respectively. Plotted are stratifications of various atmospheric parameters for the optical depth range from $\log \tau_{5}=0$ to $\log \tau_{5}=-1.8$. We differentiate between the umbral and penumbral points using continuum intensity levels. The thick solid and dotted lines show the umbral and penumbral averages respectively. The dot-dashed and dashed lines in the velocity plot show the average $v_{\text {LOS }}$ for the limb-side and center-side penumbra. The shaded areas represent the rms fluctuations in these parameters. The temperature profiles are of similar shape since they correspond to shifted 

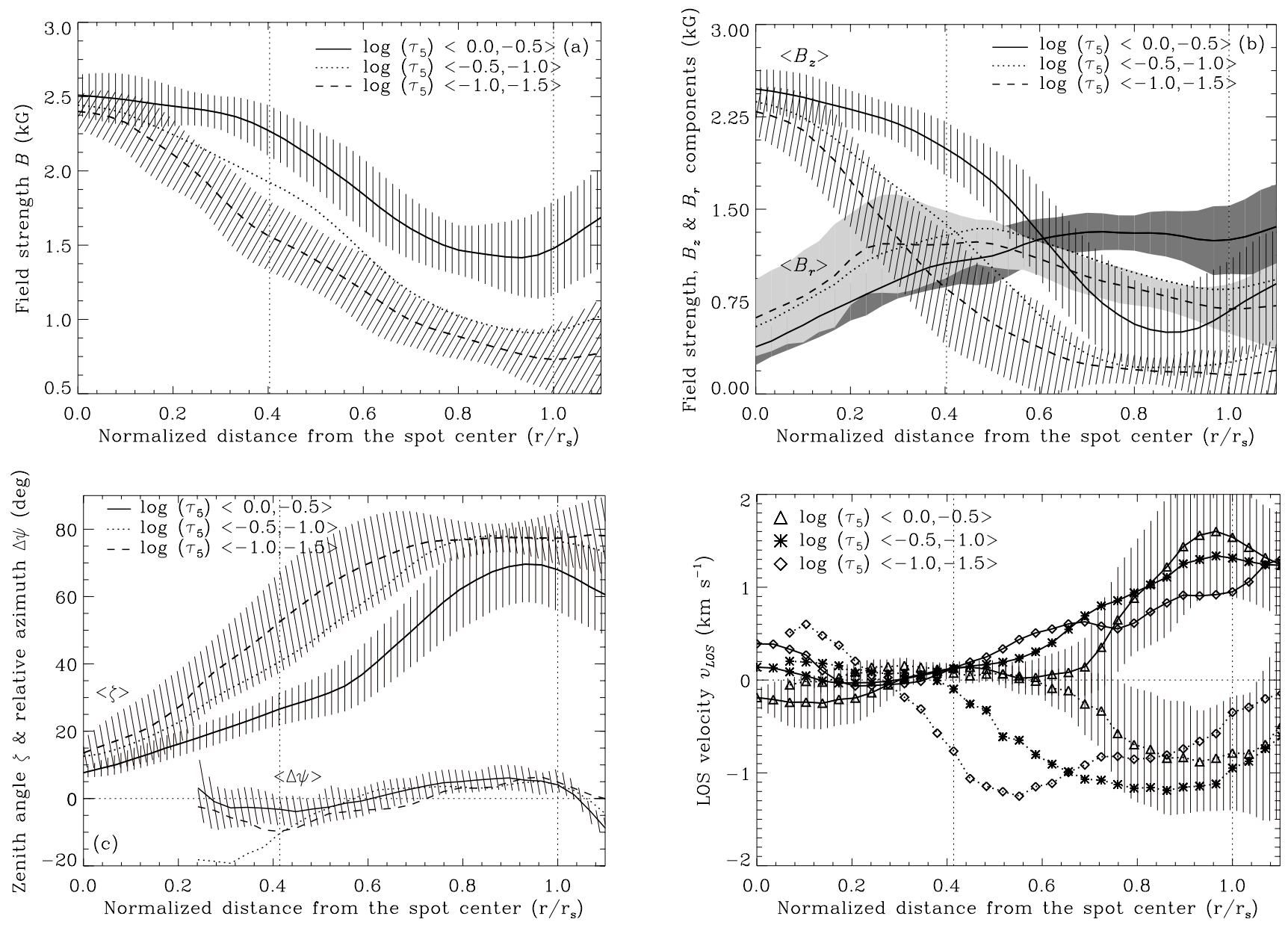

Fig. 15. The azimuthal averages for various parameters in the observed sunspot. The vertical lines represent the umbral and penumbral boundaries. The stripes/shaded areas represent the rms variation of these parameters along each radial path. For clarity the rms is indicated only for the top and bottom layers (bottom layer only for line-of-sight velocity).

Kurucz model atmospheres. The average temperature difference between umbra and penumbra is around $750 \mathrm{~K}$, while the penumbra is $200-250 \mathrm{~K}$ cooler than the quiet Sun (dotteddashed line in the temperature plot indicates the Kurucz quiet Sun model $)$. Thus we obtain $T\left(\tau_{5000}=1\right)=5500 \mathrm{~K}$ in the umbra and $T\left(\tau_{5000}=1\right)=6350 \mathrm{~K}$ in the penumbra. These values are probably too high due to the presence of stray light from the quiet Sun. The magnetic field variation with optical depth in the umbra is comparable to Collados et al. (1994) "hot" umbral model. The average field inclination varies from $20^{\circ}-30^{\circ}$ in the umbra, while in the penumbra it varies from $40^{\circ}-70^{\circ}$. In particular, in the umbra, the vertical gradient of $\zeta$ is not significant, while in the penumbra it is. Note that the large change in $\zeta$ takes place over the same height range as the large drop in field strength, between $0>\log \tau_{5}>-1$. Over this height range the average velocity amplitude also varies by around a factor of 2 on both the limb-side and center-side of the penumbra. These combined gradients may be artificial and may result from the requirement that a single magnetic component must reproduce the often strongly asymmetric profiles (see Fig. 6) caused by the complex "uncombed" magnetic structure of the

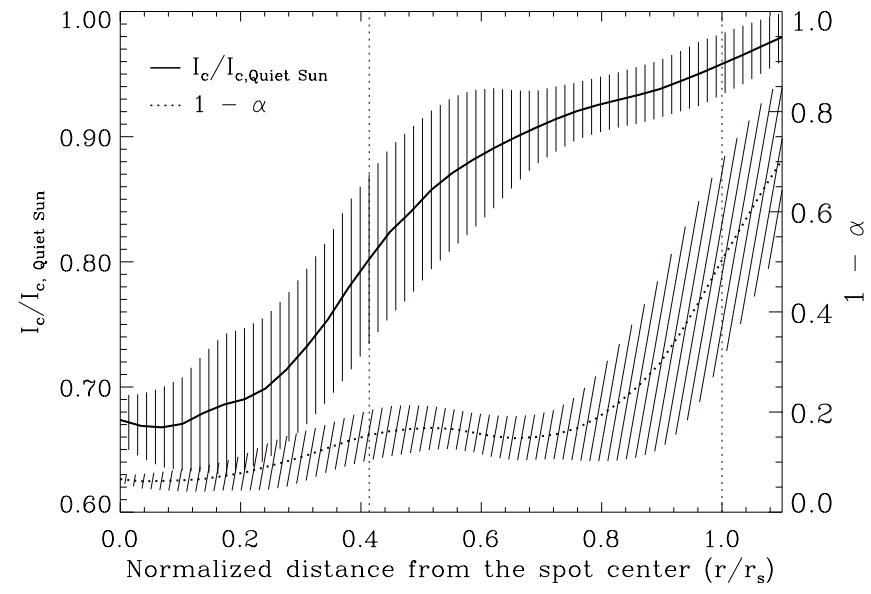

Fig. 16. Azimuthal averages of the normalized continuum intensity $\left(I / I_{\text {QuietSun }}\right)$ and scattered light parameter $(1-\alpha)$. The vertical lines represent the umbral and penumbral boundaries. The stripes represent the rms variation of these parameters.

penumbra. A clear asymmetry in average line-of-sight velocities for the limb-side and center-side penumbra is observed. This asymmetry is seen even better in Fig. 17 than in Fig. 16. 

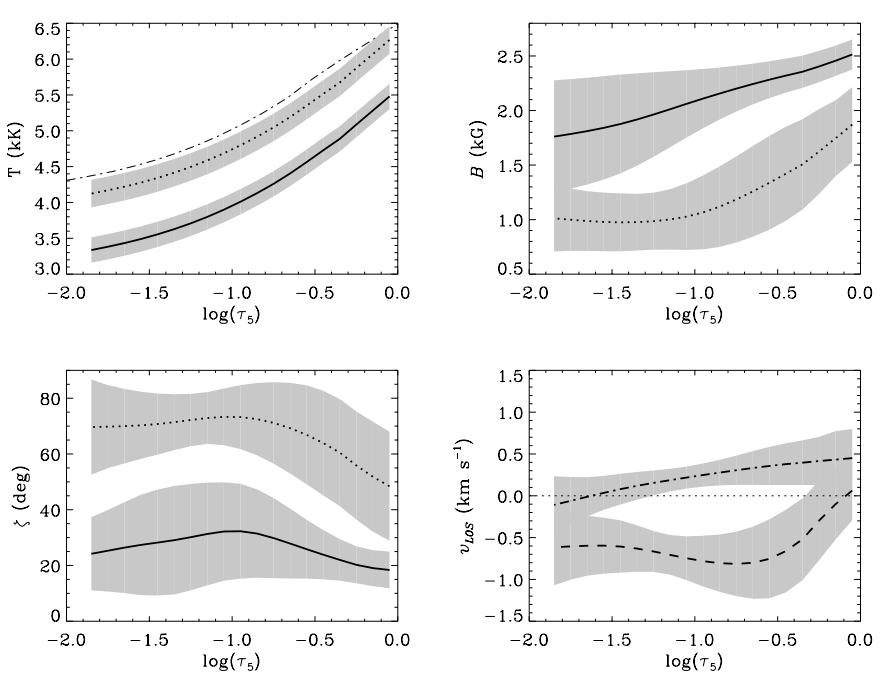

Fig. 17. Average umbral and penumbral stratification obtained from the inversion. The solid line represents the umbral average and dotted line the penumbral average. In the $v_{\text {LOS }}$ plot (lower right frame) the dot-dashed and dashed line represent the limb-side and center-side average velocities. The dot-dashed line in the temperature plot represents the Kurucz quiet Sun model.

\section{Discussion and conclusion}

We have inverted a spectropolarimetric map of a relatively symmetric sunspot, observed near solar disc center in $\mathrm{Fe}_{\mathrm{I}}$ lines around $1.56 \mu \mathrm{m}$. As a result of our inversion, we obtain the atmospheric stratification of various physical parameters within the sunspot. In the following we discuss our results and compare them with earlier investigations.

The $\mathrm{Fe}_{\mathrm{I}}$ lines used in our observations are complementary to more commonly used visible lines (e.g. Fe I $6302 \AA$ ) in a number of ways. The IR lines posses a much higher sensitivity to the magnetic field than their counterparts in the visible, allowing a more reliable determination of the magnetic vector. The IR lines are also formed deep in the photosphere, and thus are more sensitive to variations of physical parameters there. On the other hand they are far less sensitive to the upper photosphere and in fact provide reliable information only up to $150-200 \mathrm{~km}$ above the $\tau=1$ level. They are also relatively temperature insensitive (and both lines have nearly the same temperature sensitivity), which has both advantages (less temperature cross-talk to other parameters) and disadvantages (poor temperature diagnostics). The combined temperature insensitivity of these lines and of the continuum intensity at $1.6 \mu \mathrm{m}$ also means that the atmospheric parameters deduced by these lines sample contributions from cooler and brighter parts of sunspots almost equally. Finally, the Fe I $15652.8 \AA$ line is blended by $\mathrm{OH}$ lines in the umbra. Again this has disadvantages (reduced reliability there) and advantages (good temperature diagnostics, if the blending lines are modeled and fitted).

There exist few published results, where these lines are used for magnetic field measurements in sunspots (e.g. Kopp \& Rabin 1992; McPherson et al. 1992; Solanki et al. 1992b). The results reported by these authors are basically limited to few slices through the sunspot, and only height-independent information has been obtained from them. Thus, since our inversion results provide the atmospheric stratification of the parameters, it is now possible to compare results obtained from IR data with similar findings obtained using visible lines (e.g. Westendorp Plaza et al. 2001a,b; cf. Bellot Rubio 2003).

The overall structure of the radial dependence of the field strength (Fig. 15) is similar to results reported earlier. In the lower layers, the maximum field found in the umbral core of the analyzed sunspot is around $2800 \mathrm{G}\left(B_{0}\right)$. In the penumbral boundary an average value of around $1500 \mathrm{G}\left(B\left(r_{\mathrm{p}}\right)\right)$ is found. This gives a $B\left(r_{\mathrm{p}}\right) / B_{0}$ of around 0.5 for the lower layers. In higher layers, this ratio drops to around 0.3. Averaged over the range $\log \tau_{5}=0.0$ to -1.5 we obtain a ratio of 0.4 which lies somewhat below the upper limit of the values reported earlier (Lites et al. 1990; Solanki et al. 1992b; Keppens \& Martínez Pillet 1996; see the overview by Solanki 2003).

In our results we found a decreasing magnetic field with increasing height all over the sunspot right out to its continuum boundary. This disagrees with the recent results reported by Westendorp Plaza et al. (2001a). They used visible Fe I lines (6301.5 $\AA$ and $6302.5 \AA$ ) observed by the Advanced Stokes Polarimeter (ASP, Elmore et al. 1992) for their study. They found an increasing field strength with height over the line formation height range in the outer penumbra. Martínez Pillet (2000) has argued that the application of an inversion based on a one-component model to a multi component atmosphere including horizontal flux tubes embedded in an inclined field can return such gradients, even if the field strength does not actually increase with height. Since in our case the inversions were also carried out with a single magnetic component, we should in principle also observe a similar effect. We believe, however, that the difference in the results could be due to the different sensitivity of the observed lines to the various atmospheric layers. Consider the explanation by Martínez Pillet (2000), which is based on the uncombed penumbral model proposed by Solanki \& Montavon (1993). It involves a horizontal flux tube with a diameter of $100-200 \mathrm{~km}$, located at a fixed height in the photosphere, say $150 \mathrm{~km}$ above $\tau_{1.6}=1$ and embedded in a more vertical magnetic field. The emergent spectra from such a model could in fact be interpreted by inversion codes in a different way (i.e. retrieving different atmospheric stratifications) when visible lines (formed in higher layers) or infrared lines (formed in deeper layers) are inverted separately using a one component model.

In order to clarify this, we have carried out test inversions of Stokes profiles synthesized using a two component model atmosphere, in which one component describes the characteristics of the embedded flux tube and the other the background penumbral field. We introduced a flux tube of $100 \mathrm{~km}$ diameter at a height of $150 \mathrm{~km}$ above the $\tau_{5}=1$ level, in the same way as described in Borrero et al. (2002). Stokes profiles were then calculated for Fe I $6301.5 \AA$ and $6302.5 \AA$ lines in the visible and $\mathrm{Fe}_{\mathrm{I}} 15648.5 \AA$ and $15652.8 \AA$ lines in the infrared.

The test results are shown in Fig. 18. The dotted and dotteddashed lines in the three top panels show the model parameters used for the synthesis. The synthetic profiles produced using this two-component model atmosphere are shown by the solid lines in the bottom panels. The dots in these plots 

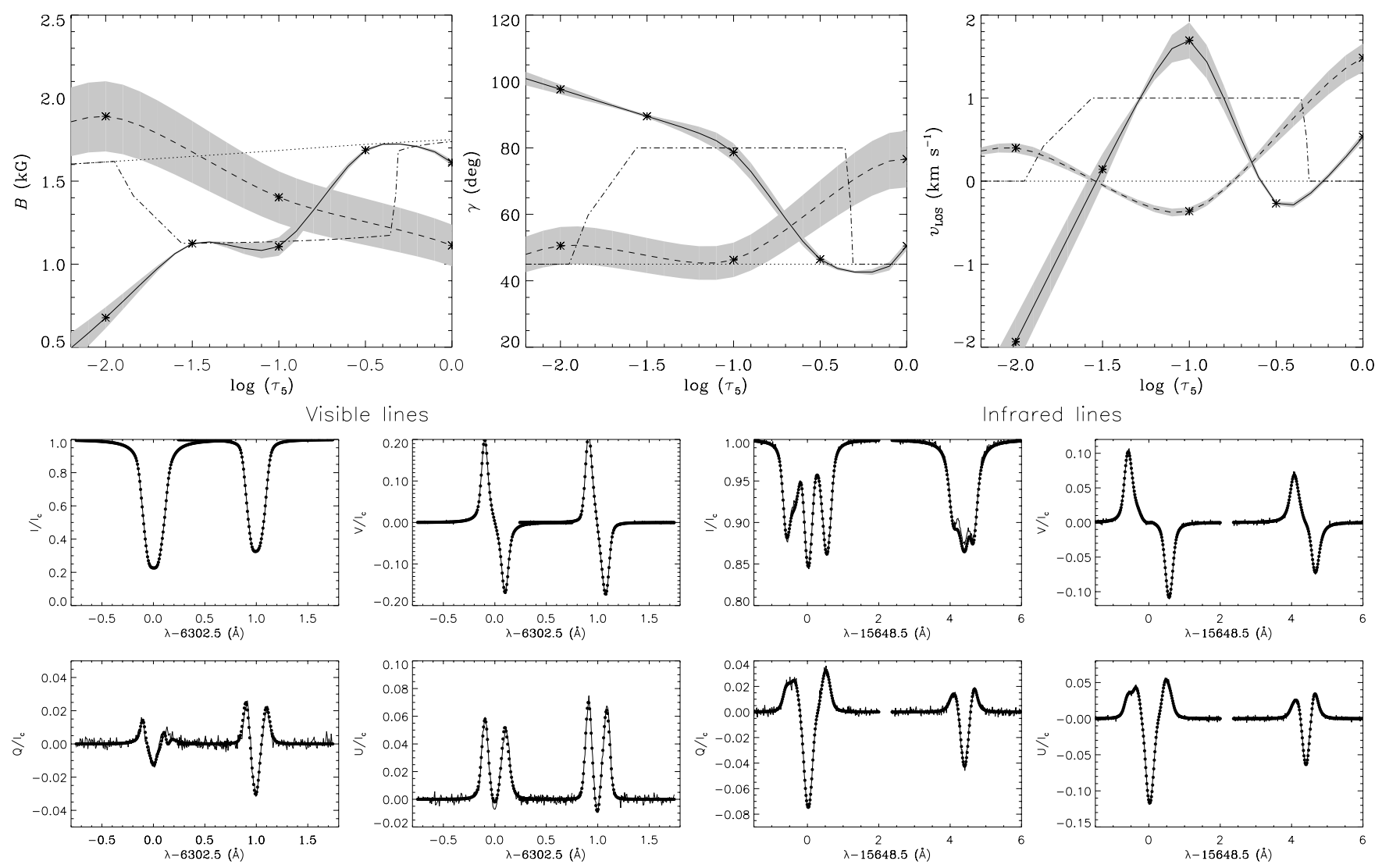

Fig. 18. Test inversion of visible and infrared lines, computed using a two magnetic component flux-tube model atmosphere. Solid curves and dots in the bottom-eight frames display the synthetic profiles and the fits obtained from the single magnetic component inversions. Top three frames show the two component flux-tube model (dot-dashed curves - the flux-tube model, dotted curves - the background) used for the synthesis, and the returned atmospheres for visible (dashed curves) and infrared lines (solid curves) when inverted with a single magnetic component. The shaded area represents the standard deviation of these parameters for inversions starting from different initial values. The asterisks symbols show the locations of nodes used in the inversions.

represent the fits obtained for these lines by inverting the profiles using a single magnetic component to describe the atmosphere. The retrieved atmosphere from the single magnetic component inversion is shown by the solid and dashed lines in the top three frames. The shaded area represents the standard deviation of these parameters for inversions starting from different initial values. The dashed lines represent the atmospheric parameters retrieved from the inversion of $6302.5 \AA$ and $6301.5 \AA$ lines whereas the solid lines represents the same for $15648.5 \AA$ and $15652.8 \AA$ spectral lines. As expected, the inversion of the visible lines returns an increasing field strength with height, while the IR lines retrieve a decreasing field strength with height. In the case of field inclination $(\gamma)$ the visible lines retrieve a decreasing inclination with height whereas the IR lines returns $\gamma$ increasing with height. In a similar study by Bellot Rubio et al. (2002) using the same IR lines also obtained an increasing $\gamma$ with height, which strengthens our results. A similar opposite behavior is also seen in the retrieved line-of-sight velocities. The difference between our results and those obtained by Westendorp Plaza et al. (2001a) is thus well reproduced by the model of Solanki \& Montavon (1993).

Our inversion results allow us to calculate the vertical field gradient in the observed sunspot. We obtain a fairly large vertical field-strength gradient of on average $-4 \mathrm{G} \mathrm{km}^{-1}$ in the umbra and the penumbra. Even though this is in agreement with the value reported by Collados et al. (1994, cf. Bellot Rubio et al. 2001) for their "hot" umbra, it is found to be larger than most of the results reported earlier (e.g., Pahlke \& Wiehr 1990; Balthasar \& Schmidt 1993). Thus, Westendorp Plaza et al. (2001a) found a value of -1.5 to $-2 \mathrm{G} \mathrm{km}^{-1}$ for the vertical field gradient in the umbra. We must stress that in the penumbra, the large gradient is limited to deeper layers, not well sampled by the lines in the visible. Note that the large gradients may be an artefact of an inversion based on a model with just a single magnetic component.

In the $B_{z}$ map we found clear signatures of "spine" structures, which are similar to the ones described by Lites et al. (1993). These structures are locations of stronger and more vertical fields than their surroundings. We also observe a small azimuthal angle change in these structures, as described by Lites et al. (1993). In lower layers we could follow this spine structures up to and beyond the penumbral boundary, whereas in the upper layers these structures are more diffuse.

There are a limited number of locations showing magnetic return flux. Such fields are only visible in the lowest observable layers near the outer edge of the penumbra, in good agreement with the results of 1-component inversions of 
Westendorp Plaza et al. (1997b). The presence of return flux in the central penumbra, as deduced by Del Toro Iniesta et al. (2001) from an inversion with a 2-component model, could not be confirmed, but may require a more sophisticated model to detect (a theoretical case for such down-flows has been made by e.g. Schlichenmaier \& Solanki 2003).

We find that the strongest magnetic field has the most vertical orientation. The inclination steadily increases outward from the umbra, and reaches a value of around $70^{\circ}-80^{\circ}$ in the outer penumbra, which is consistent with earlier observations (Lites \& Skumanich 1990; Solanki et al. 1992b; Lites et al. 1993; Keppens \& Martínez Pillet 1996; Westendorp Plaza et al. 2001a). Along the vertical direction the inclination increases with height, which again disagrees with the results obtained by Westendorp Plaza et al. (2001a). They obtain a decreasing inclination with increasing height. This disagreement can be explained by the difference in formation heights of the lines as demonstrated by the test calculations described above (see Fig. 18). The spine structures in $\zeta$ maps is characterized by lower zenith angles. The field azimuth angle is found to vary relatively smoothly along the azimuthal direction, without showing significant twist.

Regarding the absence of a canopy (as mentioned in Sect. 4.1), it is clear that beyond the visible boundary of the penumbra one should take into account many different structures like: canopy, moving magnetic features and small-scale magnetic elements piercing the canopy, intranetwork fields and the underlying quiet sun. These can only been investigated by means of more complex models since inversions based on one component models can hardly provide a coherent physical picture. Further studies should also include many lines able to sample different photospheric layers and better spatial resolution.

In the velocity map it is clear that the Evershed flow avoids the vertical spine structures, and mostly follows the more inclined field between the spine structures. In lower layers, correlation coefficients of -0.3 and 0.7 for $B-\left|v_{\text {LOS }}\right|$ and $\zeta-\left|v_{\text {LOS }}\right|$ are found for an azimuthal path at 0.8 penumbral radii, with $B$ and $\zeta$ being correlated at the 0.6 level. In the lower layers we find that the strongest flows in the velocity map coincide with those locations in the outer penumbra, where the vertical field component changes sign, i.e. locations of return flux $\left(\zeta>90^{\circ}\right)$. Presumably, these represent down-flowing material. The magnetic return flux in the middle penumbra has recently been confirmed by Bellot Rubio et al. (2003), who also show that the Evershed flow is parallel to the magnetic field lines. Although the velocity pattern changes significantly with height, the peak values remain relatively similar. Hence the marked increase of Evershed flow with depth, deduced from lines in the visible (Del Toro Iniesta et al. 1994; Westendorp Plaza et al. 2001b) does not appear to extend to the lowest part of the atmosphere sampled by the IR lines.

Acknowledgements. We would like to thank the referee L. R. Bellot Rubio for useful suggestions.

\section{References}

Ballesteros, E., Collados, M., Bonet, J. A., et al. 1996, A\&AS, 115, 353

Balthasar, H., \& Schmidt, W. 1993, A\&A, 279, 243

Bellot Rubio, L. R. 2003, in 3rd International workshop on solar polarization, ed. J. Trujillo Bueno, \& J. Sánchez Almeida, ASP Conf. Ser., in press

Bellot Rubio, L. R., Collados, M., Ruiz Cobo, B., \& Rodríguez Hidalgo, I. 2000, ApJ, 534, 989

Bellot Rubio, L. R., Rodríguez Hidalgo, I., Collados, M., Khomenko, E., \& Ruiz Cobo, B. 2001, ApJ, 560, 1010

Bellot Rubio, L. R., Collados, M., Ruiz Cobo, B., \& Rodríguez Hidalgo, I. 2002, Il Nuovo Cimento, 25, 543

Bellot Rubio, L. R., Balthasar, H., Collados, M., \& Schlichenmaier, R. 2003, A\&A, 403, L47

Berdyugina, S. V., \& Solanki, S. K. 2001, A\&A, 380, L5

Berdyugina, S. V., Solanki, S. K., \& Lagg, A. 2002, in Cool Stars, Stellar Systems and the Sun XII, ed. T. Ayres, ASP Conf. Ser., in press

Borrero, J. M., Lagg, A., Solanki, S. K., et al. 2003, in Current Theoretical Models and High Resolution Solar Observations: Preparing for ATST, ed. A. A. Pevtsov, \& H. Uitenbroek, ASP Conf. Ser., 286, 235

Bruls, J. H. M. J., Lites, B. W., \& Murphy, G. A. 1991, in Solar polarimetry, Proc. 11th Sacramento Peak Workshop, ed. L. November, 444

Choudhuri, A. R. 1986, ApJ, 302, 809

Collados, M. 1999, in Magnetic field and oscillations, ed. B. Schmieder, B. Hofmann, \& J. Staude, ASP Conf. Ser., 184, 3

Collados, M. 2003, in Polarimetry in Astronomy, ed. Silvano Fineschi, Proc. SPIE, 4843, 55

Collados, M., Martínez Pillet, V., Ruiz Cobo, B., Del Toro Iniesta, J. C., \& Vázquez, M. 1994, A\&A, 291, 622

Collados, M., Joven, E., Fuentes, F. J., et al. 1997, in Advances in the Physics of Sunspots, ed. B. Schmieder, J. C. Del Toro Iniesta, \& M. Vázquez, ASP Conf. Ser., 118, 361

Degenhardt, D., \& Wiehr, E. 1991, A\&A, 252, 821

Degenhardt, D., \& Lites, B. W. 1993a, ApJ, 404, 383

Degenhardt, D., \& Lites, B. W. 1993b, ApJ, 416, 875

Del Toro Iniesta, J. C, Tarbell, T. D., \& Ruiz Cobo, B. 1994, ApJ, 436, 400

Del Toro Iniesta, J. C., Bellot Rubio, L. R., \& Collados, M. 2001, A\&A, 549, L139

Elmore, D. F., Lites, B. W., Tomczyk, S., et al. 1992, Proc. SPIE, 1746, 22

Frutiger, C. 2000, Inversion of Zeeman split Stokes profiles: Application to solar and stellar surface structures, Ph.D. Thesis, Institute of Astronomy, ETH Zürich, No. 13896

Frutiger, C., Solanki, S. K., Fligge, M., \& Bruls, J. H. M. J. 2000, A\&A, 358, 1109

Hagyard, M. J. 1987, Sol. Phys., 107, 239

Harvey, J. W. 1977, in Highlights of Astronomy, ed. E. A. Müller, 4, 223

Keppens, R., \& Martínez Pillet, V. 1996, A\&A, 316, 229

Khomenko, E. V., Collados, M., Solanki, S. K., Lagg, A., \& Trujillo Bueno, J. 2003, A\&A, 408, 1115

Kopp, G., \& Rabin, D. 1992, Sol. Phys., 141, 253

Kurucz, R. L. 1992, in The Stellar Population of Galaxies, ed. B. Barbuy, \& A. Renzini, IAU Symp., 149, 225

Landi Degl'Innocenti, E., \& Landolfi, M. 1982, Sol. Phys., 77, 13

Lin, H. 1995, ApJ, 446, L421

Lin, H., \& Rimmele, T. 1999, ApJ, 514, 448

Lites, B. W., \& Skumanich, A. P. 1990, ApJ, 348, 747 
Lites, B. W., Elmore, D. F., Seagraves, P., \& Skumanich, A. P. 1993, ApJ, 418, 928

Livingston, W. 1991, BAAS, 23, 1030

Livingston, W. 2002, Sol. Phys., 207, 41

Martínez Pillet, V. 1997, 1st Advances in Solar Physics Euroconference. Advances in Physics of Sunspots, ASP Conf. Ser., 118, 212

Martínez Pillet, V. 2000, A\&A, 361, 374

Martínez Pillet, V. 2001, A\&A, 369, 644

Martínez Pillet, V., Collados, M., Sánchez Almeida, J., et al. 1999, in High Resolution Solar Physics: Theory, Observations, and Techniques, ed. T. R. Rimmele, K. S. Balasubramaniam, \& R. R. Radick, ASP Conf. Ser., 183, 264

McPherson, M. R., Lin, H., \& Kuhn, J. R. 1992, Sol. Phys., 139, 255

Muglach, K., \& Solanki, S. K. 1992, A\&A, 263, 301

Pahlke, K. D., \& Wiehr, E. 1990, A\&A, 228, 246

Parker, E. N. 1979, ApJ, 234, 333

Press, W. H., Teukolsky, S. A., Vetterling, W. T., \& Flannery, B. P. 1992, Numerical Recipes (New-York: Cambridge Univ. Press)

Ruiz Cobo, B., \& Del Toro Iniesta, J. C. 1992, ApJ, 398, 375

Ruiz Cobo, B., \& Del Toro Iniesta, J. C. 1994, A\&A, 283, 129

Rüedi, I., Solanki, S. K., Livingston, W., \& Stenflo, J. O. 1992a, A\&A, 263,323

Rüedi, I., Solanki, S. K., \& Rabin, D. 1992b, A\&A, 261, L21

Rüedi, I., Solanki, S. K., Keller, C. U., \& Frutiger, C. 1998, A\&A, 338,1089

Rüedi, I., Solanki, S. K., \& Keller, C. U. 1999, A\&A, 348, L37

Sánchez Almeida, J., \& Lites, B. W. 1992, ApJ, 398, 359
Schlichenmaier, R., \& Schmidt, W. 2000, A\&A, 358, 1122

Schlichenmaier, R., \& Collados, M. 2002, A\&A, 381, 668

Schlichenmaier, R., \& Solanki, S. K. 2003, A\&A, submitted

Schmidt, W., Hofmann, A., Balthasar, H., Tarbell, T. D., \& Frank, Z. A. 1992, A\&A, 264, L27

Skumanich, A., \& Lites, B. W. 1987, ApJ, 322, 473

Solanki, S. K. 1987, Photspheric layers of solar magnetic fluxtubes, Ph.D. Thesis, Institute of Astronomy, ETH Zürich, No. 8309

Solanki, S. K. 2003, A\&ARv, 11, 153

Solanki, S. K., \& Montavon, C. A. P. 1993, A\&A, 275, 283

Solanki, S. K., Rüedi, I., \& Livingston, W. 1992a, A\&A, 263, 312

Solanki, S. K., Rüedi, I., \& Livingston, W. 1992b, A\&A, 263, 339

Solanki, S. K., Walther, U., \& Livingston, W. 1993, A\&A, 277, 639

Solanki, S. K., Zufferey, D., Lin, H., Rüedi, I., \& Kuhn, J. R. 1996, A\&A, 310, L33

Sun, W.-H., Giampapa, M. S., \& Worden, S. P. 1987, ApJ, 312, 930

Title, A. M., Frank, Z. A., Shine, R. A., et al. 1993, ApJ, 403, 780

Venkatakrishnan, P., Hagyard, M. J., \& Hathaway, D. H. 1988, Sol. Phys., 115, 25

Westendorp Plaza, C., Del Toro Iniesta, J. C., Ruiz Cobo, B., et al. 1997a, Nature, 389, 47

Westendorp Plaza, C., Del Toro Iniesta, J. C., Ruiz Cobo, B., et al. 1997b, in Advances in the Physics of Sunspots, ed. B. Schmieder, J. C. Del Toro Iniesta, \& M. Vázquez, ASP Conf. Ser., 118, 202

Westendorp Plaza, C., Del Toro Iniesta, J. C., Ruiz Cobo, B., et al. 2001a, ApJ, 547, 1130

Westendorp Plaza, C., Del Toro Iniesta, J. C., Ruiz Cobo, B., et al. 2001b, ApJ, 547, 1148 